\title{
An Approach to the Core Skeleton of Lancifodilactone F
}

\author{
Qiaoling Wang and Chuo Chen
}

\section{Experimental Section}

General Procedures. All reactions were performed in glassware under a positive pressure of argon. Liquids and solvent were transferred via syringe. Organic solutions were concentrated by rotary evaporator at ca. $10 \mathrm{mmHg}$. Flash column chromatography was performed as described by Still (Still, W. C.; Kahn, M.; Mitra, A. J. Org. Chem. 1978, 43, 2923-2925), employing EMD silica gel 60 (230400 mesh ASTM), or using a HPFC Biotage system (Biotage, Inc.) with pre-packed FLASH silica gel. TLC analyses were performed on EMD $250 \mu \mathrm{m}$ Silica Gel $60 \mathrm{~F}_{254}$ plates and visualized by quenching of UV fluorescence $\left(\lambda_{\max }=254 \mathrm{~nm}\right)$, or by staining with ceric ammonium molybdate or potassium permanganate. ${ }^{1} \mathrm{H}$ and ${ }^{13} \mathrm{C}$ NMR spectra were recorded on a Varian Inova-500, Inova-400 or Mercury300 spectrometer. Chemical shifts for ${ }^{1} \mathrm{H}$ and ${ }^{13} \mathrm{C}$ NMR spectra are reported in ppm $(\delta)$ relative to residue protium in the solvent $\left(\mathrm{CDCl}_{3}: \delta 7.26,77.0 \mathrm{ppm} ; \mathrm{CD}_{3} \mathrm{OD}: \delta 3.31,49.0 \mathrm{ppm}\right.$; benzene- $d_{6}: \delta$ $7.16,128.4 \mathrm{ppm}$ ) and the multiplicities are presented as follows: $\mathrm{s}=$ singlet, $\mathrm{d}=$ doublet, $\mathrm{t}=$ triplet, $\mathrm{m}$ $=$ multiplet, $\mathrm{br}=$ broad. Infrared spectra were recorded on a Perkin-Elmer 1000 series FTIR. Mass spectrometry was provided by the Washington University Mass Spectrometry Resource with support from the NIH National Center for Research Resources (Grant No. P41RR0954).

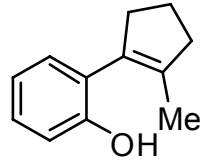

2-(2-Methylcyclopent-1-enyl)phenol (13). To a solution of 2-bromophenol (11) (3.46 g, $20.0 \mathrm{mmol}, 1.0$ equiv) in diethyl ether $(80 \mathrm{~mL})$ cooled at $0{ }^{\circ} \mathrm{C}$ was added $n$-butyllithium (2.5 $\mathrm{M}$ in hexanes, $16.0 \mathrm{~mL}, 40.0 \mathrm{mmol}, 2.0$ equiv). After stirring for $3 \mathrm{~h}$, the solution was cooled to $-78^{\circ} \mathrm{C}$ and 2-methylcyclopentanone (12) (1.96 g, $20.0 \mathrm{mmol}, 1.0$ equiv) was added. The reaction was slowly warmed to $23{ }^{\circ} \mathrm{C}$ and stirred for another $3 \mathrm{~h}$ before concentrated hydrochloric acid $(20 \mathrm{~mL})$ was added. After stirring for $1 \mathrm{~h}$, water $(5 \mathrm{~mL})$ was added. The layers were separated and the organic layer was washed with water $(5 \mathrm{~mL})$, saturated aqueous sodium bicarbonate $(15 \mathrm{~mL})$, brine $(15 \mathrm{~mL})$, dried over anhydrous sodium sulfate, filtered, concentrated, and purified by silica gel column chromatography to give 13 as colorless oil $(2.50 \mathrm{~g}, 72 \%): R_{f}=0.40(10 \%$ ethyl acetate-hexanes); FTIR (neat, $\mathrm{cm}^{-1}$ ) 2842, 2341, 1487, 1211, 1177, 752; ${ }^{1} \mathrm{H} \mathrm{NMR}\left(500 \mathrm{MHz}, \mathrm{CDCl}_{3}\right) \delta$ 7.18-7.15 (t, $J=7.7 \mathrm{~Hz}, 1 \mathrm{H}), 7.07-7.06(\mathrm{~d}, J=8.1 \mathrm{~Hz}, 1 \mathrm{H}), 6.94-6.93(\mathrm{~d}, J=7.5 \mathrm{~Hz}, 1 \mathrm{H}), 6.90-6.88(\mathrm{t}$, $J=7.5 \mathrm{~Hz}, 1 \mathrm{H}), 5.16(\mathrm{br},-\mathrm{OH}), 2.67-2.64(\mathrm{~m}, 2 \mathrm{H}), 2.54-2.51(\mathrm{t}, J=7.3 \mathrm{~Hz}, 2 \mathrm{H}), 2.00-1.95$ (dd, $J$ $=15.0,7.0 \mathrm{~Hz}, 2 \mathrm{H}), 1.65(\mathrm{~s}, 3 \mathrm{H}) ;{ }^{13} \mathrm{C} \mathrm{NMR}\left(75 \mathrm{MHz}, \mathrm{CDCl}_{3}\right) \delta 152.0,138.8,131.2,128.5,128.2$,

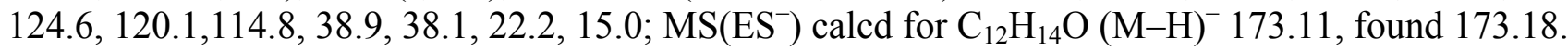

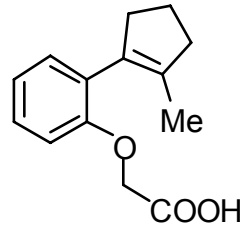

2-(2-(2-Methylcyclopent-1-enyl)phenoxy)acetic acid (14). To a solution of $\mathbf{1 3}$ (2.24 g, 12.9 mmol, 1.0 equiv) in tetrahydrofuran $(20 \mathrm{~mL})$ was added sodium hydride $(60 \%$ dispersion in mineral oil, $1.13 \mathrm{~g}, 28.3 \mathrm{mmol}, 2.2$ equiv) at $23{ }^{\circ} \mathrm{C}$. After stirring for 30 min, chloroacetic acid ( $1.19 \mathrm{~g}, 12.9 \mathrm{mmol}, 1.0$ equiv) was added. The reaction solution was then heated at $65^{\circ} \mathrm{C}$ for $5 \mathrm{~h}$ before quenching with brine $(5 \mathrm{~mL})$. Water $(5 \mathrm{~mL})$ was added to dissolve all the salts. The aqueous layer was adjusted to $\mathrm{pH} 10.5$ with $\mathrm{Na}_{2} \mathrm{CO}_{3}$ solution $(10 \mathrm{~mL})$. The aqueous layer was separated, washed twice with diethyl ether $(10 \mathrm{~mL})$, acidified to $\mathrm{pH} 1$ with concentrated hydrochloric acid $(5 \mathrm{~mL})$, and extracted with diethyl ether $(20$ $\mathrm{mL} \times 3)$. The combined extracts were washed with water $(5 \mathrm{~mL})$ and brine $(5 \mathrm{~mL})$, dried over anhydrous sodium sulfate, and evaporated in vacuo to give 14 (2.89 g, 97\%): $R_{f}=0.17$ (50\% ethyl acetate-hexanes); FTIR (neat, $\left.\mathrm{cm}^{-1}\right)$ 2925, 1726, 1487, 1444, 1202, 1122, 751; ${ }^{1} \mathrm{H}$ NMR (500 MHz, $\left.\mathrm{CD}_{3} \mathrm{OD}\right) \delta 7.17(\mathrm{t}, J=7.0 \mathrm{~Hz}, 1 \mathrm{H}), 7.07(\mathrm{~d}, J=7.5 \mathrm{~Hz}, 1 \mathrm{H}), 6.94(\mathrm{t}, J=7.4 \mathrm{~Hz}, 1 \mathrm{H}), 6.85(\mathrm{~d}, J=8.2$ $\mathrm{Hz}, 1 \mathrm{H}), 5.12(\mathrm{br},-\mathrm{OH}), 4.56(\mathrm{~s}, 2 \mathrm{H}), 2.73-2.70(\mathrm{~m}, 2 \mathrm{H}), 2.44-2.41(\mathrm{t}, J=7.0 \mathrm{~Hz}, 2 \mathrm{H}), 1.90(\mathrm{dt}, J=$ 
15.0, 8.0 Hz, 2H), $1.62(\mathrm{~s}, 3 \mathrm{H}) ;{ }^{13} \mathrm{C}$ NMR $\left(75 \mathrm{MHz}, \mathrm{CD}_{3} \mathrm{OD}\right) \delta 172.8,157.1,136.9,134.8,131.7$, 129.9, 128.8, 122.4, 113.8, 66.5, 39.7, 38.6, 23.6, 15.5; $\mathrm{MS}\left(\mathrm{ES}^{+}\right)$calcd for $\mathrm{C}_{14} \mathrm{H}_{17} \mathrm{O}_{3}(\mathrm{M}+\mathrm{H})^{+} 233.12$, found 233.1.

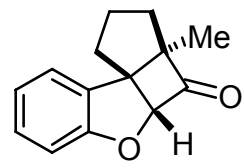

$\left(1 S^{*}, 5 R^{*}, 7 R^{*}\right)-5$-Methyl-benzofuro[2,3-g]bicycle[3.2.0]hept-6-one (15). To a solution of 14 (2.58 g, $11.1 \mathrm{mmol}, 1.0$ equiv) in toluene $(30 \mathrm{~mL})$ was added oxalyl chloride (1.94 mL, $22.2 \mathrm{mmol}, 2.0$ equiv). After heating at $110^{\circ} \mathrm{C}$ for $1.5 \mathrm{~h}$, the solution was cooled to $23{ }^{\circ} \mathrm{C}$ and volatiles were evaporated in vacuo. The residue was taken up in toluene $(20 \mathrm{~mL} \times 2)$ and evaporated in vacuo to remove excess oxalyl chloride. The resulting acid chloride was then dissolved toluene $(3 \mathrm{~mL})$ and to a solution of triethylamine $(2.32 \mathrm{~mL}$, $16.7 \mathrm{mmol}, 1.5$ equiv) in refluxing toluene $(20 \mathrm{~mL})$. After heating at $110^{\circ} \mathrm{C}$ for an additional $1.5 \mathrm{~h}$, the solution was cooled, and poured into saturated ammonium chloride solution $(20 \mathrm{~mL})$. The layers were separated and the organic phase was washed with saturated ammonium chloride solution $(10 \mathrm{~mL})$ and brine $(10 \mathrm{~mL})$, dried over anhydrous sodium sulfate, filtered, concentrated, and purified by silica gel column chromatography to give 15 as colorless oil $(1.83 \mathrm{~g}, 77 \%): R_{f}=0.40(10 \%$ ethyl acetatehexanes); FTIR (neat, $\mathrm{cm}^{-1}$ ) 2957, 2360, 1777, 1459, 1211, 956, 751; ${ }^{1} \mathrm{H} \mathrm{NMR}\left(500 \mathrm{MHz}, \mathrm{CDCl}_{3}\right) \delta$ $7.20(\mathrm{t}, J=7.8 \mathrm{~Hz}, 1 \mathrm{H}), 7.15(\mathrm{~d}, J=7.5 \mathrm{~Hz}, 1 \mathrm{H}), 6.98(\mathrm{t}, J=7.5 \mathrm{~Hz}, 1 \mathrm{H}), 6.88(\mathrm{~d}, J=8.1 \mathrm{~Hz}, 1 \mathrm{H})$, $5.25(\mathrm{~s}, 1 \mathrm{H}), 2.33-2.21(\mathrm{~m}, 3 \mathrm{H}), 2.11-2.05(\mathrm{dt}, J=13.0,6.7 \mathrm{~Hz}, 1 \mathrm{H}), 1.84-1.74(\mathrm{~m}, 1 \mathrm{H}), 1.71-1.65(\mathrm{~m}$, $1 \mathrm{H}), 0.94(\mathrm{~s}, 3 \mathrm{H}) ;{ }^{13} \mathrm{C} \mathrm{NMR}\left(75 \mathrm{MHz}, \mathrm{CDCl}_{3}\right) \delta 211.3,161.8,128.9,126.2,124.9,121.5,110.5,77.2$, 58.0, 37.9, 35.5, 26.2, 16.7; $\mathrm{MS}\left(\mathrm{ES}^{+}\right)$calcd for $\mathrm{C}_{14} \mathrm{H}_{15} \mathrm{O}_{2}(\mathrm{M}+\mathrm{H})^{+} 215.11$, found 215.1.

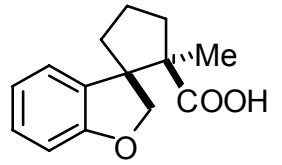

$\left(1 ' R^{*}, 2^{\prime} R^{*}\right)$-2'-Methyl-2H-spiro[benzofuran-3,1'-cyclopentane]-2'-carboxylic acid (16). To a solution of 15 (2.24 g, $10.5 \mathrm{mmol}, 1.0$ equiv) in tert-butyl alcohol $(20 \mathrm{~mL})$ was added water $(0.19 \mathrm{~mL}, 10.5 \mathrm{mmol}, 1.0$ equiv) and potassium tertbutoxide ( $2.34 \mathrm{~g}, 20.9 \mathrm{mmol}, 2.0$ equiv). The reaction was stirred at $23{ }^{\circ} \mathrm{C}$ for 30 min before quenching with $1 \mathrm{~N}$ hydrochloric acid $(10 \mathrm{~mL})$. The solution was diluted with diethyl ether $(200 \mathrm{~mL})$, washed with saturated aqueous sodium bicarbonate $(10 \mathrm{~mL})$, brine $(10 \mathrm{~mL})$, dried over anhydrous sodium sulfate, filtered and concentrated to give 16 (2.38 g, 98\%): $R_{f}=0.20$ (30\% ethyl acetate-hexanes); FTIR (neat, $\mathrm{cm}^{-1}$ ) 2968, 2879, 1691, 1479, 1234, 976, 752; ${ }^{1} \mathrm{H}$ NMR (500 MHz, $\left.\mathrm{CDCl}_{3}\right) \delta 7.19-7.14(\mathrm{~m}, 2 \mathrm{H}), 6.91(\mathrm{t}, J=7.4 \mathrm{~Hz}, 1 \mathrm{H}), 6.81(\mathrm{~d}, J=8.0 \mathrm{~Hz}, 1 \mathrm{H}), 4.70(\mathrm{~d}, J=9.6 \mathrm{~Hz}, 1 \mathrm{H})$, $4.29(\mathrm{~d}, J=9.6 \mathrm{~Hz}, 1 \mathrm{H}), 2.43-2.37(\mathrm{~m}, 1 \mathrm{H}), 2.25-2.19(\mathrm{~m}, 1 \mathrm{H}), 2.15-2.09(\mathrm{~m}, 1 \mathrm{H}), 1.96-1.89(\mathrm{~m}, 3 \mathrm{H})$, $1.15(\mathrm{~s}, 3 \mathrm{H}) ;{ }^{13} \mathrm{C} \mathrm{NMR}\left(75 \mathrm{MHz}, \mathrm{CDCl}_{3}\right) \delta 182.5,160.3,131.0,128.6,124.6,120.3,109.8,79.7,59.0$, 55.1, 37.6, 35.7, 20.4, 19.9; $\mathrm{MS}\left(\mathrm{ES}^{+}\right)$calcd for $\mathrm{C}_{14} \mathrm{H}_{17} \mathrm{O}_{3}(\mathrm{M}+\mathrm{H})^{+}$233.12, found 233.1.

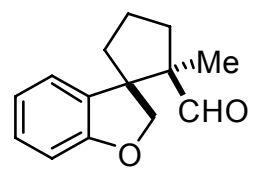

$\left(1 ' R^{*}, 2\right.$ ' $\left.R^{*}\right)$-2'-Methyl-2H-spiro[benzofuran-3,1'-cyclopentane]-2'carboxaldehyde (17). To a solution of 16 (2.20 g, $9.48 \mathrm{mmol}, 1.0$ equiv) in tetrahydrofuran $(50 \mathrm{~mL})$ was added lithium aluminum hydride $(396 \mathrm{mg}, 10.4 \mathrm{mmol}$, 1.1 equiv). The reaction was stirred at $23{ }^{\circ} \mathrm{C}$ for $30 \mathrm{~min}$ before quenching with water $(0.4 \mathrm{~mL}), 15 \%$ sodium hydroxide $(0.4 \mathrm{~mL})$ and water $(1.2 \mathrm{~mL})$. The solution was decanted, dried over anhydrous sodium sulfate, filtered, and concentrated to give alcohol $\mathbf{1 7 s}$ as colorless oil (1.82 g, 88\%): $R_{f}=0.26$ (30\% ethyl acetate-hexanes); FTIR (neat, $\mathrm{cm}^{-1}$ ) 3401, 2957, 2876, 1591, 1480, 1457, 1236, 1021, 970, 752; ${ }^{1} \mathrm{H}$ NMR $\left(500 \mathrm{MHz}, \mathrm{CDCl}_{3}\right) \delta 7.16-7.13(\mathrm{~m}, 2 \mathrm{H}), 6.91(\mathrm{t}, J=7.5 \mathrm{~Hz}, 1 \mathrm{H}), 6.82(\mathrm{~d}, J=$ $7.9 \mathrm{~Hz}, 1 \mathrm{H}), 4.78(\mathrm{~d}, J=9.0 \mathrm{~Hz}, 1 \mathrm{H}), 4.06(\mathrm{~d}, J=9.1 \mathrm{~Hz}, 1 \mathrm{H}), 3.53(\mathrm{~d}, J=11.4 \mathrm{~Hz}, 1 \mathrm{H}), 3.45(\mathrm{~d}, J=$ $11.4 \mathrm{~Hz}, 1 \mathrm{H}), 2.35-2.30(\mathrm{~m}, 1 \mathrm{H}), 1.92-1.79(\mathrm{~m}, 3 \mathrm{H}), 1.76-1.63(\mathrm{~m}, 2 \mathrm{H}), 0.92(\mathrm{~s}, 3 \mathrm{H}) ;{ }^{13} \mathrm{C}$ NMR $(75$ $\left.\mathrm{MHz}, \mathrm{CDCl}_{3}\right) \delta 160.2,131.9,128.4,125.3,120.6,110.0,80.1,68.5,58.0,48.9,36.8,34.3,19.9,19.8$; $\mathrm{MS}\left(\mathrm{ES}^{+}\right)$calcd for $\mathrm{C}_{14} \mathrm{H}_{19} \mathrm{O}_{2}(\mathrm{M}+\mathrm{H})^{+} 219.14$, found 219.1 .

To a solution of the above obtained alcohol ( $1.70 \mathrm{~g}, 7.80 \mathrm{mmol}, 1.0$ equiv) in methylene chloride (50 $\mathrm{mL}$ ) cooled at $-78^{\circ} \mathrm{C}$ was added dimethyl sulfoxide (1.65 mL, $23.4 \mathrm{mmol}, 3.0$ equiv) and oxalyl 
chloride (1.36 mL, $15.6 \mathrm{mmol}, 2.0$ equiv). After stirring at $-78^{\circ} \mathrm{C}$ for $30 \mathrm{~min}$, triethylamine $(3.26 \mathrm{~mL}$, $23.4 \mathrm{mmol}, 4.0$ equiv) was added. The reaction was then warmed to $23{ }^{\circ} \mathrm{C}$ and stirred for an additional $30 \mathrm{~min}$ before quenching with $1 \mathrm{~N}$ hydrochloric acid $(10 \mathrm{~mL})$. The layers were separated and the organic layer was washed with saturated aqueous sodium bicarbonate $(10 \mathrm{~mL})$, brine $(10 \mathrm{~mL})$, dried over anhydrous sodium sulfate, filtered, concentrated, and purified by silica gel column chromatography to give 17 as colorless oil (1.53 g, 91\%): $R_{f}=0.57$ (30\% ethyl acetate-hexanes); FTIR (neat, $\mathrm{cm}^{-1}$ ) 2961, 2877, 1718, 1593, 1480, 1458, 1235, 974, 754; ${ }^{1} \mathrm{H}$ NMR $\left(500 \mathrm{MHz}, \mathrm{CDCl}_{3}\right) \delta 9.47$ $(\mathrm{s}, 1 \mathrm{H}), 7.19-7.16(\mathrm{~m}, 2 \mathrm{H}), 6.93(\mathrm{t}, J=7.4 \mathrm{~Hz}, 1 \mathrm{H}), 6.82(\mathrm{~d}, J=8.0 \mathrm{~Hz}, 1 \mathrm{H}), 4.56(\mathrm{~d}, J=9.6 \mathrm{~Hz}, 1 \mathrm{H})$, $4.26(\mathrm{~d}, J=9.6 \mathrm{~Hz}, 1 \mathrm{H}), 2.29-2.23(\mathrm{~m}, 2 \mathrm{H}), 2.01-1.93(\mathrm{~m}, 2 \mathrm{H}), 1.85-1.73(\mathrm{~m}, 2 \mathrm{H}), 1.02(\mathrm{~s}, 3 \mathrm{H}) ;{ }^{13} \mathrm{C}$ $\operatorname{NMR}\left(75 \mathrm{MHz}, \mathrm{CDCl}_{3}\right) \delta 204.3,160.0,130.8,128.7,124.7,120.5,110.0,78.8,58.8,58.0,36.7,31.9$, 36.7, 31.9, 20.2, 16.9; $\mathrm{MS}\left(\mathrm{ES}^{+}\right)$calcd for $\mathrm{C}_{14} \mathrm{H}_{17} \mathrm{O}_{2}(\mathrm{M}+\mathrm{H})^{+}$217.12, found 217.1.

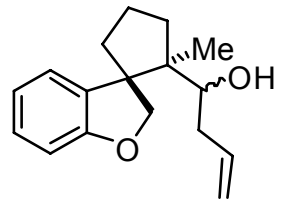

(1' $\left.S^{*}, 2^{\prime} R^{*}\right)-2$ '-Methyl-2'-(1-hydroxybut-3-enyl)-2H-spiro[benzofuran-3,1'cyclopentane] (18). To a solution of 17 (1.34 g, $6.20 \mathrm{mmol}, 1.0$ equiv) in tetrahydrofuran $(40 \mathrm{~mL})$ cooled at $-78^{\circ} \mathrm{C}$ was added allylmagnesium bromide $(1.0$ $\mathrm{M}$ in diethyl ether, $6.50 \mathrm{~mL}, 6.50 \mathrm{mmol}, 1.05$ equiv). After stirring for $10 \mathrm{~min}$, the reaction was quenched with $1 \mathrm{~N}$ hydrochloric acid $(5 \mathrm{~mL})$. The layers were

separated and the organic layer was washed with saturated aqueous sodium bicarbonate $(10 \mathrm{~mL})$, brine $(10 \mathrm{~mL})$, dried over anhydrous sodium sulfate, filtered, concentrated, and purified by silica gel column chromatography to give 18a (744 mg) and 18b (744 mg) as colorless oil (93\%).

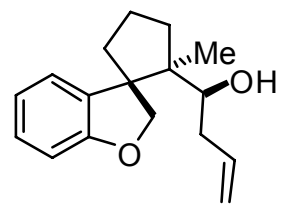

18a: $R_{f}=0.37\left(10 \%\right.$ ethyl acetate-hexanes); FTIR (neat, $\left.\mathrm{cm}^{-1}\right) 3596,2956,2878$, 1593, 1481, 1457, 1233, 989, 752; ${ }^{1} \mathrm{H}$ NMR (500 MHz, $\left.\mathrm{CDCl}_{3}\right) \delta 7.21(\mathrm{~d}, J=7.3 \mathrm{~Hz}$, $1 \mathrm{H}), 7.13(\mathrm{t}, J=7.7 \mathrm{~Hz}, 1 \mathrm{H}), 6.90(\mathrm{t}, J=7.3 \mathrm{~Hz}, 1 \mathrm{H}), 6.80(\mathrm{~d}, J=8.0 \mathrm{~Hz}, 1 \mathrm{H}), 5.89-$ $5.81(\mathrm{~m}, 1 \mathrm{H}), 5.06-5.03(\mathrm{~m}, 2 \mathrm{H}), 4.87(\mathrm{~d}, J=9.3 \mathrm{~Hz}, 1 \mathrm{H}), 4.07(\mathrm{~d}, J=9.3 \mathrm{~Hz}, 1 \mathrm{H})$, $3.93(\mathrm{dd}, J=8.5,4.1 \mathrm{~Hz}, 1 \mathrm{H}), 2.50-2.43(\mathrm{~m}, 1 \mathrm{H}), 2.11-2.08(\mathrm{~m}, 2 \mathrm{H}), 1.87-1.81(\mathrm{~m}$, $1 \mathrm{H}), 1.78-1.73(\mathrm{~m}, 1 \mathrm{H}), 1.68-1.51(\mathrm{~m}, 4 \mathrm{H}), 1.04(\mathrm{~s}, 3 \mathrm{H}) ;{ }^{13} \mathrm{C} \mathrm{NMR}\left(75 \mathrm{MHz}, \mathrm{CDCl}_{3}\right) \delta 160.2,136.4$, 131.7, 128.4, 125.0, 120.6, 117.0, 109.8, 80.9, 75.2, 58.3, 51.4, 38.0, 37.6, 35.6, 19.4, 15.8; $\mathrm{MS}_{\left(\mathrm{ES}^{+}\right)}$ calcd for $\mathrm{C}_{17} \mathrm{H}_{23} \mathrm{O}_{2}(\mathrm{M}+\mathrm{H})^{+} 259.17$, found 259.0 .

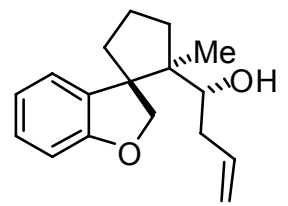

18b: $R_{f}=0.20$ (10\% ethyl acetate-hexanes); FTIR (neat, $\left.\mathrm{cm}^{-1}\right) 3467,2958,2878$, 1593, 1481, 1458, 1236, 975, 752; ${ }^{1} \mathrm{H}$ NMR (500 MHz, $\left.\mathrm{CDCl}_{3}\right) \delta 7.17-7.13(\mathrm{~m}, 2$ $\mathrm{H}), 6.90(\mathrm{t}, J=7.5 \mathrm{~Hz}, 1 \mathrm{H}), 6.80(\mathrm{~d}, J=7.9 \mathrm{~Hz}, 1 \mathrm{H}), 5.78-5.69(\mathrm{~m}, 1 \mathrm{H}), 5.05-4.99$ $(\mathrm{m}, 2 \mathrm{H}), 4.90(\mathrm{~d}, J=9.2 \mathrm{~Hz}, 1 \mathrm{H}), 4.05(\mathrm{~d}, J=9.2 \mathrm{~Hz}, 1 \mathrm{H}), 3.65(\mathrm{dd}, J=9.4,2.6 \mathrm{~Hz}$, $1 \mathrm{H}), 2.46-2.40(\mathrm{~m}, 1 \mathrm{H}), 1.94-1.61(\mathrm{~m}, 7 \mathrm{H}), 0.93(\mathrm{~s}, 3 \mathrm{H}) ;{ }^{13} \mathrm{C} \mathrm{NMR}(75 \mathrm{MHz}$, $\left.\mathrm{CDCl}_{3}\right) \delta 160.6,136.3,131.3,128.3,125.1,120.3,117.7,109.7,80.9,74.5,58.0,51.0,38.0,37.7,34.9$, 19.1, 16.6; $\mathrm{MS}\left(\mathrm{ES}^{+}\right)$calcd for $\mathrm{C}_{17} \mathrm{H}_{23} \mathrm{O}_{2}(\mathrm{M}+\mathrm{H})^{+}$259.17, found 259.18.

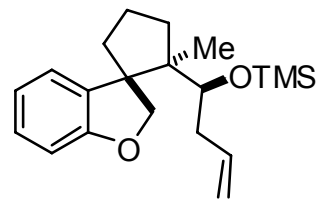

$\left(1 ' S^{*}, 2\right.$ ' $\left.R^{*}\right)-2$ '-Methyl-2'-[( $\left.S^{*}\right)$-1-trimethylsiloxybut-3-enyl)-2Hspiro[benzofuran-3,1'-cyclopentane] (19). To 18a (700 mg, $2.71 \mathrm{mmol}, 1.0$ equiv) was added $N$-trimethylsilyl imidazole $(0.80 \mathrm{~mL}, 5.42 \mathrm{mmol}, 2.0$ equiv). After stirring for $5 \mathrm{~min}$, the reaction was filtered with short silica gel column to give the pure 19 as colorless oil (858 $\mathrm{mg}, 96 \%): R_{f}=0.42(2 \%$ ethyl acetate-

hexanes); FTIR (neat, $\mathrm{cm}^{-1}$ ) 2957, 2879, 1593, 1482, 1458, 1249, 1091, 839, 749; ${ }^{1} \mathrm{H}$ NMR (500 MHz, $\left.\mathrm{CDCl}_{3}\right) \delta 7.18(\mathrm{~d}, J=7.5 \mathrm{~Hz}, 1 \mathrm{H}), 7.08(\mathrm{~d}, J=7.8 \mathrm{~Hz}, 1 \mathrm{H}), 6.82(\mathrm{t}, J=7.4 \mathrm{~Hz}, 1 \mathrm{H}), 6.74(\mathrm{~d}, J=7.9$ $\mathrm{Hz}, 1 \mathrm{H}), 5.82-5.77(\mathrm{~m}, 1 \mathrm{H}), 5.04(\mathrm{~d}, J=8.9 \mathrm{~Hz}, 1 \mathrm{H}), 4.98-4.94(\mathrm{~m}, 2 \mathrm{H}), 4.03(\mathrm{~d}, J=8.9 \mathrm{~Hz}, 1 \mathrm{H})$, $3.83(\mathrm{dd}, J=6.7,4.1 \mathrm{~Hz}, 1 \mathrm{H}), 2.27-2.17(\mathrm{~m}, 2 \mathrm{H}), 2.13-2.09(\mathrm{~m}, 1 \mathrm{H}), 1.86-1.74(\mathrm{~m}, 3 \mathrm{H}), 1.70-1.64$ $(\mathrm{m}, 1 \mathrm{H}), 1.50-1.47(\mathrm{~m}, 1 \mathrm{H}), 1.15(\mathrm{~s}, 3 \mathrm{H}), 0.06(\mathrm{~s}, 9 \mathrm{H}) ;{ }^{13} \mathrm{C} \mathrm{NMR}\left(75 \mathrm{MHz}, \mathrm{CDCl}_{3}\right) \delta 160.5,137.5$, 
$132.9,127.5,124.9,119.8,116.0,109.6,80.0,77.7,57.9,52.2,40.5,39.0,36.4,19.3,19.0,0.6$; $\mathrm{MS}\left(\mathrm{ES}^{+}\right)$calcd for $\mathrm{C}_{20} \mathrm{H}_{31} \mathrm{O}_{2} \mathrm{Si}(\mathrm{M}+\mathrm{H})^{+} 331.21$, found 331.1 .

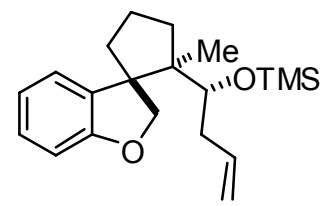

$\left(1 ' S^{*}, 2\right.$ ' $\left.R^{*}\right)-2$ '-Methyl-2'-[( $\left.R^{*}\right)$-1-trimethylsiloxybut-3-enyl)-2Hspiro[benzofuran-3,1'-cyclopentane] (20). To $\mathbf{1 8 b}(700 \mathrm{mg}, 2.71 \mathrm{mmol}, 1.0$ equiv) was added $N$-trimethylsilyl imidazole $(0.80 \mathrm{~mL}, 5.42 \mathrm{mmol}, 2.0$ equiv). After stirring for $5 \mathrm{~min}$, the reaction was filtered with short silica gel column to give the pure 20 as colorless oil $(858 \mathrm{mg}, 96 \%): R_{f}=0.38(2 \%$ ethyl acetate-

hexanes); FTIR (neat, $\mathrm{cm}^{-1}$ ) 2957, 2979, 1595, 1481, 1458, 1249, 1068, 838, 752; ${ }^{1} \mathrm{H}$ NMR (500 MHz, $\left.\mathrm{CDCl}_{3}\right) \delta 7.16-7.13(\mathrm{~m}, 2 \mathrm{H}), 6.89(\mathrm{t}, J=7.2 \mathrm{~Hz}, 1 \mathrm{H}), 6.78(\mathrm{~d}, J=7.9 \mathrm{~Hz}, 1 \mathrm{H}), 5.71-5.65(\mathrm{~m}, 1 \mathrm{H})$, $4.90(\mathrm{~d}, J=10.0 \mathrm{~Hz}, 1 \mathrm{H}), 4.79-4.75(\mathrm{~m}, 2 \mathrm{H}), 4.04$ (d, $J=9.2 \mathrm{~Hz}, 1 \mathrm{H}), 3.79$ (d, $J=7.4 \mathrm{~Hz}, 1 \mathrm{H}), 2.48-$ $2.43(\mathrm{~m}, 1 \mathrm{H}), 1.80-1.59(\mathrm{~m}, 6 \mathrm{H}), 1.28-1.24(\mathrm{~m}, 1 \mathrm{H}), 0.96(\mathrm{~s}, 3 \mathrm{H}), 0.10(\mathrm{~s}, 9 \mathrm{H}) ;{ }^{13} \mathrm{C} \mathrm{NMR}(75 \mathrm{MHz}$ $\left.\mathrm{CDCl}_{3}\right) \delta 160.7,137.3,131.4,128.3,125.1,120.2,116.2,109.6,81.0,77.7,57.4,52.5,38.7,38.4,37.5$, 18.4, 15.3, 1.0; $\mathrm{MS}\left(\mathrm{ES}^{+}\right)$calcd for $\mathrm{C}_{17} \mathrm{H}_{23} \mathrm{O}_{2}\left(\mathrm{M}-\mathrm{TMS}+\mathrm{H}_{2} \mathrm{O}\right)^{+} 259.1698$, found 259.1692 .

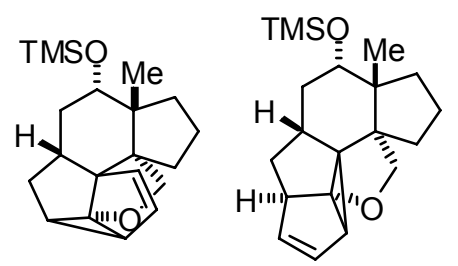

$\left(1 R^{*}, 5 R^{*}, 6 S^{*}, 8 R^{*}, 10 S^{*}, 11 S^{*}, 14 S^{*} 17 S^{*}\right)-5-M e t h y l-16-0 x a-6-$ trimethylsiloxylhexacyclo $\left[7.4 .3 .0^{1,5} \cdot 0^{8,14} \cdot 0^{11,17} \cdot 0^{14,17}\right]$ heptadec-12-ene (21) and $\left(1 R^{*}, 5 R^{*}, 6 S^{*}, 8 R^{*}, 10 R^{*}, 13 R^{*}, 14 S^{*} 17 S^{*}\right)-5$-methyl-16-oxa-6trimethylsiloxylhexacyclo $\left[7.4 .3 .0^{1,5} \cdot 0^{8,14} \cdot 0^{13,17} \cdot 0^{14,17}\right]$ heptadec-11-ene (22). A solution of 19 (100 mg, $0.30 \mathrm{mmol}, 1.0$ equiv) in cyclohexane (50 $\mathrm{mL}$ ) was degassed by bubbling argon through the solution for $30 \mathrm{~min}$.

The solution was then photolyzed at $23{ }^{\circ} \mathrm{C}$ in a Rayonet chamber reactor at $254 \mathrm{~nm}$ or by low-pressure Hanovia low pressure mercury lamp through quartz filter for $40 \mathrm{~min}$. The solution was then concentrated to give 21 and 22 (1:1.2) as colorless oil, which can be used directly for the next step. Filtrate through a short pad of silica gel gave pure 21 and 22 (1:1.5): $R_{f}=0.19$ (2\% ethyl acetatehexanes); FTIR (neat, $\mathrm{cm}^{-1}$ ) 2955, 2878, 1250, 1094, 1077, 922, 873, 839, 730; ${ }^{1} \mathrm{H} \mathrm{NMR} \mathrm{(500} \mathrm{MHz,}$ benzene- $\left.d_{6}\right) \delta 5.62(\mathrm{dd}, J=7.5,2.9 \mathrm{~Hz}, 1 \mathrm{H}), 5.59(\mathrm{dd}, J=6.0,2.5 \mathrm{~Hz}, 1 \mathrm{H}), 5.57(\mathrm{dd}, J=5.2,2.2 \mathrm{~Hz}$, $1 \mathrm{H}), 5.54-5.53(\mathrm{~m}, 1 \mathrm{H}), 5.37(\mathrm{~d}, J=5.2 \mathrm{~Hz}, 1 \mathrm{H}), 4.84(\mathrm{dd}, J=8.9,1.8 \mathrm{~Hz}, 1 \mathrm{H}), 3.76-3.74(\mathrm{~m}, 2 \mathrm{H})$, $3.66-3.63(\mathrm{~m}, 1 \mathrm{H}), 3.56(\mathrm{~d}, J=7.5 \mathrm{~Hz}, 1 \mathrm{H}), 3.28(\mathrm{dd}, J=5.2,2.9 \mathrm{~Hz}, 1 \mathrm{H}), 2.98(\mathrm{~s}, 1 \mathrm{H}), 2.67$ (ddd, $J=$ 11.2, 11.2, $5.4 \mathrm{~Hz}, 1 \mathrm{H}), 2.31(\mathrm{~d}, J=7.8 \mathrm{~Hz}, 1 \mathrm{H}), 2.14-2.04(\mathrm{~m}, 4 \mathrm{H}), 2.01-1.90(\mathrm{~m}, 7 \mathrm{H}), 1.77-1.36(\mathrm{~m}$, $8 \mathrm{H}), 1.12-1.07(\mathrm{~m}, 3 \mathrm{H}), 0.71(\mathrm{~s}, 3 \mathrm{H}), 0.68(\mathrm{~s}, 3 \mathrm{H}), 0.08(\mathrm{~s}, 9 \mathrm{H}), 0.05(\mathrm{~s}, 9 \mathrm{H}) ;{ }^{13} \mathrm{C} \mathrm{NMR}(75 \mathrm{MHz}$, benzene- $\left.d_{6}\right) \delta 134.9,134.0,129.2,127.5,97.5,97.4,89.9,82.2,75.4,74.0,70.8,58.1,57.6,54.5,54.1$, 51.1, 47.9, 47.7, 47.0, 46.7, 38.0, 34.5, 34.0, 33.2, 33.1, 32.8, 32.7, 32.2, 31.9, 30.4, 25.7, 25.2, 20.4, 20.3, 0.5, 0.3; $\mathrm{MS}\left(\mathrm{ES}^{+}\right)$calcd for $\mathrm{C}_{20} \mathrm{H}_{31} \mathrm{O}_{2} \mathrm{Si}(\mathrm{M}+\mathrm{H})^{+} 331.21$, found 331.1.

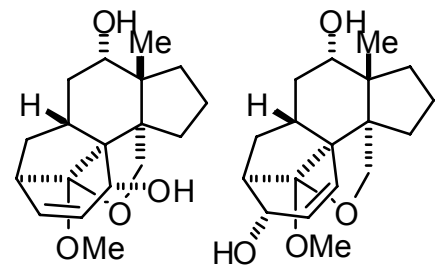

$\left(1 S^{*}, 5 R^{*}, 6 S^{*}, 8 R^{*}, 10 R^{*}, 13 R^{*}, 14 R^{*}, 17 R^{*}\right)-6,13-D i h y d r o x y l-17-$ methoxyl-5-methyl-16-oxapentacyclo $\left[7.4 .3 .0^{1,5} \cdot 0^{8,14} \cdot 0^{14,17}\right.$ heptadec-11ene $(23)$ and $\left(1 S^{*}, 5 R^{*}, 6 S^{*}, 8 R^{*}, 10 R^{*}, 11 S^{*}, 14 S^{*}, 17 R^{*}\right)-6,11-$ dihydroxyl17-methoxyl-5-methyl-16-oxapentacyclo[7.4.3.0 $\left.{ }^{1,5} \cdot 0^{8,14} \cdot 0^{14,17}\right]$ heptadec12-ene (24). To a solution of 21 and 22 (100 mg, $0.30 \mathrm{mmol}, 1.0$ equiv) in methylene chloride $(5 \mathrm{~mL})$ at $23{ }^{\circ} \mathrm{C}$ was added $m$-chloroperbenzoic acid (104 mg, $0.60 \mathrm{mmol}, 2.0$ equiv). After stirring for $1 \mathrm{~h}$, the reaction was quenched with sodium thiosulfate $(10 \%$ in saturated aqueous sodium bicarbonate, $5 \mathrm{~mL})$. The layers were separated and the organic layer was washed with saturated aqueous sodium bicarbonate $(5 \mathrm{~mL})$, brine $(5 \mathrm{~mL})$, dried over anhydrous sodium sulfate, filtered, and concentrated to give epoxides $23 \mathrm{~s}$ and 24s as white solid: $R_{f}=$ 0.19 (10\% ethyl acetate-hexanes); FTIR (neat, $\left.\mathrm{cm}^{-1}\right)$ 2956, 2879, 1250, 1057, 841; ${ }^{\mathrm{H} ~ \mathrm{NMR}}(500 \mathrm{MHz}$, benzene- $\left.d_{6}\right) \delta 5.50(\mathrm{~m}, 1 \mathrm{H}), 4.77(\mathrm{~m}, 1 \mathrm{H}), 3.76-3.71(\mathrm{~m}, 2 \mathrm{H}), 3.65-3.62(\mathrm{~m}, 1 \mathrm{H}), 3.50-3.48(\mathrm{~m}, 1 \mathrm{H})$, $3.12(\mathrm{~m}, 1 \mathrm{H}), 2.97(\mathrm{~m}, 1 \mathrm{H}), 2.83(\mathrm{~m}, 2 \mathrm{H}), 2.67(\mathrm{~m}, 2 \mathrm{H}), 2.48$ (dddd, $J=11.8,11.8,5.6,2.2 \mathrm{~Hz}, 1 \mathrm{H})$, $2.25(\mathrm{~m}, 1 \mathrm{H}), 2.18(\mathrm{~d}, J=8.7 \mathrm{~Hz}, 1 \mathrm{H}), 2.02-1.96(\mathrm{~m}, 2 \mathrm{H}), 1.87-1.80(\mathrm{~m}, 4 \mathrm{H}), 1.76-1.72(\mathrm{dd}, J=11.9$, 
$6.0 \mathrm{~Hz}, 1 \mathrm{H}), 1.67-1.56(\mathrm{~m}, 6 \mathrm{H}), 1.53-1.48(\mathrm{~m}, 3 \mathrm{H}), 1.35-1.25(\mathrm{~m}, 3 \mathrm{H}), 1.07-1.02(\mathrm{~m}, 2 \mathrm{H}), 0.70(\mathrm{~s}$, $3 \mathrm{H}), 0.64(\mathrm{~s}, 3 \mathrm{H}), 0.06(\mathrm{~s}, 9 \mathrm{H}), 0.03(\mathrm{~s}, 9 \mathrm{H}) ;{ }^{13} \mathrm{C}$ NMR $\left(75 \mathrm{MHz}\right.$, benzene- $\left.d_{6}\right) \delta$ 91.5, 89.4, 89.3, 82.7, 75.2, 73.8, 65.7, 58.4, 57.8, 57.7, 57.2, 56.1, 54.2, 53.4, 50.2, 47.3, 47.0, 46.5, 46.3, 45.4, 38.9, 33.6, $33.4,33.3,33.0,32.9,32.7,32.6,32.1,29.5,25.7,25.2,20.4,20.2,0.4,0.3 ; \mathrm{MS}_{\left(\mathrm{ES}^{+}\right)}$calcd for $\mathrm{C}_{20} \mathrm{H}_{31} \mathrm{O}_{3} \mathrm{Si}(\mathrm{M}+\mathrm{H})^{+} 347.20$, found 347.1.

To a solution of epoxides $23 \mathrm{~s}$ and $24 \mathrm{~s}$ (104 $\mathrm{mg}, 0.30 \mathrm{mmol}, 1.0$ equiv) in methanol was added $p$ toluenesulfonic acid (11.5 mg, $0.60 \mathrm{mmol}, 2.0$ equiv) at $23{ }^{\circ} \mathrm{C}$. After stirring for $5 \mathrm{~min}$, the reaction was quenched with saturated aqueous sodium bicarbonate $(5 \mathrm{~mL})$. The mixture was extracted with ethyl acetate $(3 \times 10 \mathrm{~mL})$ and the combined organic layers were washed with saturated aqueous sodium bicarbonate $(5 \mathrm{~mL})$, brine $(5 \mathrm{~mL})$, dried over anhydrous sodium sulfate, filtered, concentrated, and purified by silica gel column chromatography to give $\mathbf{2 3}$ (29 mg, 31\% over three steps) and 24 (35 mg, $38 \%$ over three steps) as white solid. Recrystallization of $\mathbf{2 3}$ from diethyl ether gave colorless prisms suitable for single X-ray crystallographic analysis (CCDC 659037).

23: $R_{f}=0.33$ (50\% ethyl acetate-hexanes); FTIR (neat, $\left.\mathrm{cm}^{-1}\right) 3448,2953,2881,1060,1022 ;{ }^{1} \mathrm{H}$ NMR $\left(500 \mathrm{MHz}\right.$, benzene- $\left.d_{6}\right) \delta 5.77(\mathrm{dd}, J=9.4,4.1 \mathrm{~Hz}, 1 \mathrm{H}), 5.65(\mathrm{dd}, J=9.4,6.8 \mathrm{~Hz}, 1 \mathrm{H}), 4.47(\mathrm{~d}, J=8.7$ $\mathrm{Hz}, 1 \mathrm{H}), 4.14(\mathrm{~d}, J=3.9 \mathrm{~Hz}, 1 \mathrm{H}), 3.63(\mathrm{~d}, J=8.7 \mathrm{~Hz}, 1 \mathrm{H}), 3.35-3.25(\mathrm{~m}, 2 \mathrm{H}), 2.98(\mathrm{~s}, 3 \mathrm{H}), 2.54(\mathrm{dd}, J$ $=6.3,5.8 \mathrm{~Hz}, 1 \mathrm{H}), 2.12(\mathrm{ddd}, J=14.3,8.7,5.8 \mathrm{~Hz}, 1 \mathrm{H}), 1.89-1.75(\mathrm{~m}, 2 \mathrm{H}), 1.74-1.64(\mathrm{~m}, 2 \mathrm{H}), 1.55-$ 1.49 (m, 2H), 1.42-1.36 (m, 2H), 1.06 (ddd, $J=12.5,9.4,3.2 \mathrm{~Hz}, 1 \mathrm{H}), 0.85(\mathrm{~s}, 3 \mathrm{H}) ;{ }^{13} \mathrm{C} \mathrm{NMR}(75$ MHz, benzene- $\left.d_{6}\right) \delta 130.5,127.8,116.6,79.8,77.8,73.0,57.8,55.8,50.4,48.0,41.4,41.0,39.0,36.8$, 36.3, 31.6, 28.6, 20.3; MS(EI) calcd for $\mathrm{C}_{18} \mathrm{H}_{26} \mathrm{O}_{4}(\mathrm{M})^{+} 306$, found 306 .

24: $R_{f}=0.24\left(50 \%\right.$ ethyl acetate-hexanes); FTIR (neat, $\left.\mathrm{cm}^{-1}\right) 3427,2960,2880,1066,998 ;{ }^{1} \mathrm{H}$ NMR $\left(500 \mathrm{MHz}\right.$, benzene- $\left.d_{6}\right) \delta 5.88(\mathrm{~d}, J=9.6 \mathrm{~Hz}, 1 \mathrm{H}), 5.72(\mathrm{ddd}, J=9.7,3.6,1.3 \mathrm{~Hz}, 1 \mathrm{H}), 4.58(\mathrm{~d}, J=8.9$ $\mathrm{Hz}, 1 \mathrm{H}), 3.98(\mathrm{dt}, J=11.6,2.8 \mathrm{~Hz}, 1 \mathrm{H}), 3.50(\mathrm{~d}, J=8.9 \mathrm{~Hz}, 1 \mathrm{H}), 3.35(\mathrm{t}, J=8.7 \mathrm{~Hz}, 1 \mathrm{H}), 3.10(\mathrm{~s}, 3 \mathrm{H})$, 2.72-2.68 (m, 2H), 2.00-1.34 (m, 8H), 1.05-0.90 (m, 2H), $0.58(\mathrm{~s}, 3 \mathrm{H}) ;{ }^{13} \mathrm{C} \mathrm{NMR}\left(75 \mathrm{MHz}, \mathrm{CD}_{3} \mathrm{OD}\right)$ $\delta 136.0,128.9,116.6,81.3,76.7,73.8,57.0,55.5,50.2,49.7,44.9,43.5,41.7,34.9,34.0,32.9,27.3$, 20.5 .
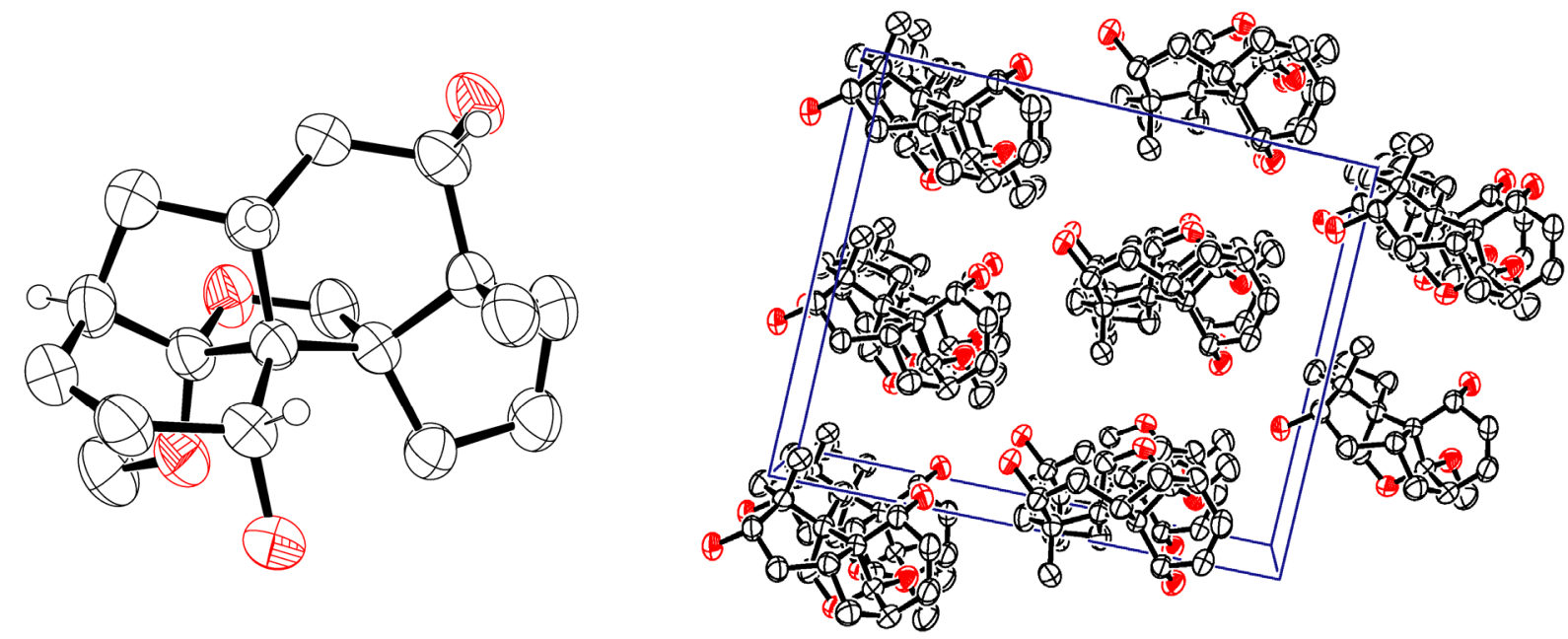

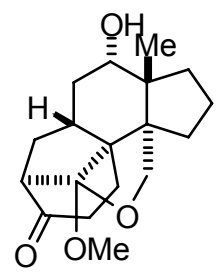

$\left(1 S^{*}, 5 R^{*}, 6 R^{*}, 8 R^{*}, 10 R^{*}, 14 R^{*}, 17 R^{*}\right)-6-H y d r o x y l-17-m e t h o x y l-5-m e t h y l-11-0 x 0-16-$ oxapentacyclo[7.4.3.0 ${ }^{1,5} \cdot 0^{8,14} \cdot 0^{14,17}$ ]heptadecane (25). To a solution of $\mathbf{2 4}$ ( $10.0 \mathrm{mg}$, $0.03 \mathrm{mmol}, 1.0$ equiv) in methol was add palladium on carbon $(30 \% \mathrm{w} / \mathrm{w}, 1.0 \mathrm{mg}) \mathrm{was}$ added and the solution was purged with hydrogen for $5 \mathrm{~min}$. After stirring for an additional $30 \mathrm{~min}$, the reaction was filted through a pad of Celite and concentrated to 
give 25 as white solid (9.7 mg, 97\%). Recrystallization from diethyl ether gave colorless prisms suitable for single X-ray crystallographic analysis (CCDC 659035): $R_{f}=0.26(50 \%$ ethyl acetatehexanes); FTIR (neat, $\mathrm{cm}^{-1}$ ) 3503, 2962, 1709, 1457, 1079; ${ }^{1} \mathrm{H}$ NMR (500 MHz, benzene- $\left.d_{6}\right) \delta 4.55(\mathrm{~d}$, $J=8.8 \mathrm{~Hz}, 1 \mathrm{H}), 3.52(\mathrm{~d}, J=8.8 \mathrm{~Hz}, 1 \mathrm{H}), 3.30(\mathrm{t}, J=8.4 \mathrm{~Hz}, 1 \mathrm{H}), 3.22(\mathrm{~s}, 3 \mathrm{H}), 3.21(\mathrm{~d}, J=7.3 \mathrm{~Hz}$,

$1 \mathrm{H}), 2.17-2.14(\mathrm{~m}, 1 \mathrm{H}), 2.03-1.95(\mathrm{~m}, 3 \mathrm{H}), 1.71-1.46(\mathrm{~m}, 8 \mathrm{H}), 1.37-1.33(\mathrm{~m}, 1 \mathrm{H}), 1.08-1.05(\mathrm{~m}, 1 \mathrm{H})$, $0.98-0.94(\mathrm{~m}, 1 \mathrm{H}), 0.49(\mathrm{~s}, 3 \mathrm{H}) ;{ }^{13} \mathrm{C}$ NMR $\left(75 \mathrm{MHz}\right.$, benzene- $\left.d_{6}\right) \delta 210.3,115.5,79.4,71.9,54.5,53.6$, 52.8, 48.6, 46.7, 40.3, 36.9, 32.9, 32.0, 31.7, 30.3, 26.4, 25.5, 18.5; $\mathrm{MS}\left(\mathrm{ES}^{+}\right)$calcd for $\mathrm{C}_{18} \mathrm{H}_{27} \mathrm{O}_{4}$ $(\mathrm{M}+\mathrm{H})^{+}$307.19, found 307.00.
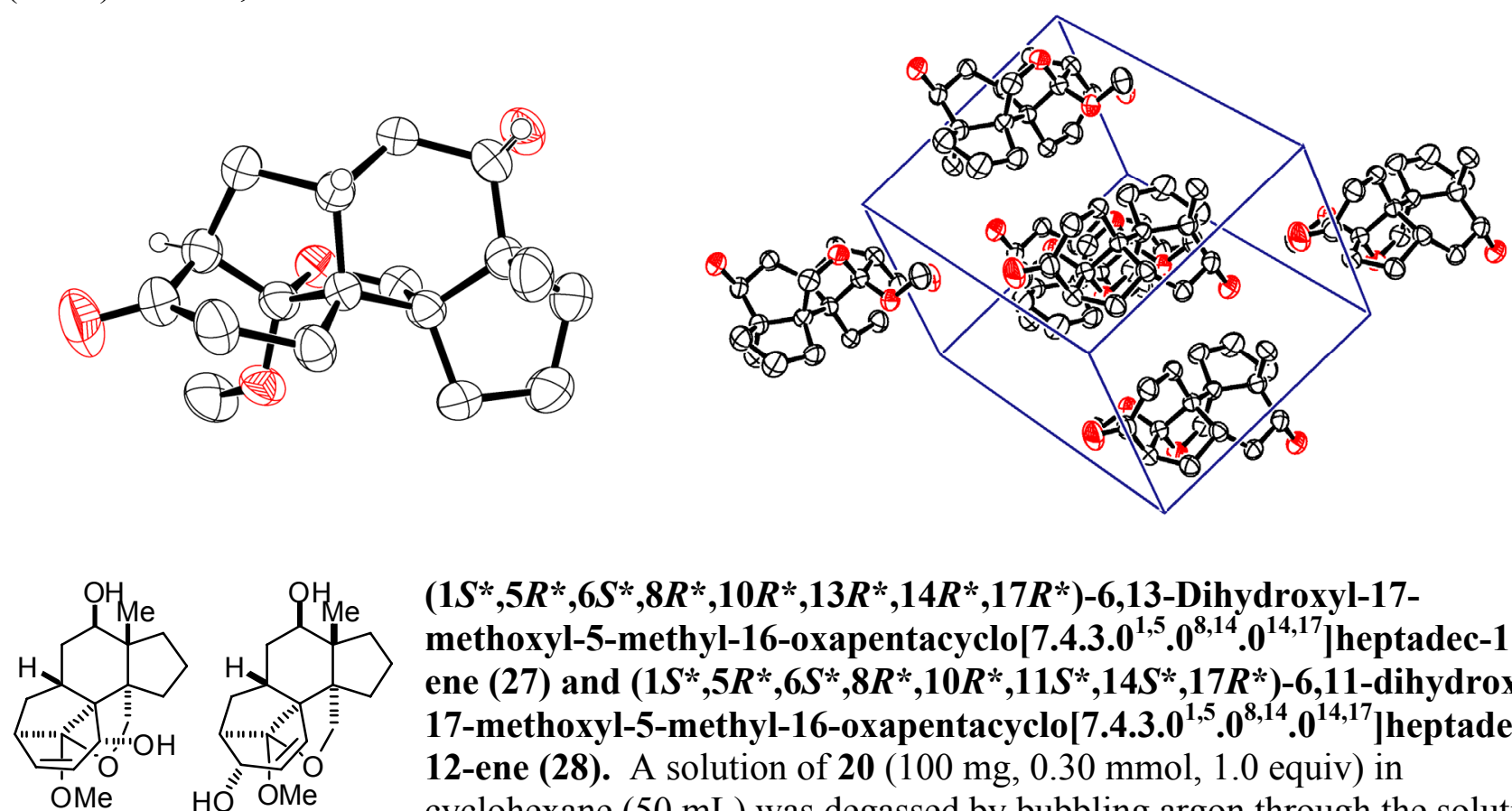

$\left(1 S^{*}, 5 R^{*}, 6 S^{*}, 8 R^{*}, 10 R^{*}, 13 R^{*}, 14 R^{*}, 17 R^{*}\right)-6,13-\mathrm{Dihydroxyl}-17-$ methoxyl-5-methyl-16-oxapentacyclo[7.4.3.0 ${ }^{1,5} \cdot 0^{8,14} \cdot 0^{14,17}$ heptadec-11ene $(27)$ and $\left(1 S^{*}, 5 R^{*}, 6 S^{*}, 8 R^{*}, 10 R^{*}, 11 S^{*}, 14 S^{*}, 17 R^{*}\right)-6,11-$ dihydroxyl17-methoxyl-5-methyl-16-oxapentacyclo $\left[7.4 .3 .0^{1,5} \cdot 0^{8,14} \cdot 0^{14,17}\right]$ heptadec12-ene (28). A solution of 20 (100 $\mathrm{mg}, 0.30 \mathrm{mmol}, 1.0$ equiv) in cyclohexane $(50 \mathrm{~mL})$ was degassed by bubbling argon through the solution for $30 \mathrm{~min}$. The solution was then photolyzed at $23{ }^{\circ} \mathrm{C}$ in a Rayonet chamber reactor at $254 \mathrm{~nm}$ or by low-pressure Hanovia low pressure mercury lamp through quartz filter for $60 \mathrm{~min}$. The solution was then concentrated to give 27s1 and 28s1: $R_{f}=0.18$ ( $2 \%$ ethyl acetate-hexanes) FTIR (neat, $\left.\mathrm{cm}^{-1}\right) 2956$, $2878,1249,1077,922,874,838,735 ;{ }^{1} \mathrm{H}$ NMR $\left(500 \mathrm{MHz}\right.$, benzene- $\left.d_{6}\right) \delta 5.51-5.50(\mathrm{~m}, 2 \mathrm{H}), 5.46-45$ $(\mathrm{m}, 1 \mathrm{H}), 5.38(\mathrm{~d}, J=5.3 \mathrm{~Hz}, 1 \mathrm{H}), 4.47(\mathrm{~d}, J=7.7 \mathrm{~Hz}, 1 \mathrm{H}), 4.21(\mathrm{dd}, J=10.1,6.2 \mathrm{~Hz}, 1 \mathrm{H}), 3.99(\mathrm{~d}, J=$ $8.7 \mathrm{~Hz}, 1 \mathrm{H}), 3.88(\mathrm{~d}, J=8.2 \mathrm{~Hz}, 1 \mathrm{H}), 3.58(\mathrm{~d}, J=9.6 \mathrm{~Hz}, 1 \mathrm{H}), 3.40$ (dd, $J=7.5,1.1 \mathrm{~Hz}, 1 \mathrm{H}), 3.19$ (s, $1 \mathrm{H}), 2.89(\mathrm{~s}, 1 \mathrm{H}), 2.33-2.30(\mathrm{~m}, 1 \mathrm{H}), 2.27(\mathrm{~d}, J=7.9 \mathrm{~Hz}, 1 \mathrm{H}), 2.18-1.99(\mathrm{~m}, 4 \mathrm{H}), 1.82-1.31(\mathrm{~m}, 16 \mathrm{H})$, $0.99(\mathrm{~s}, 3 \mathrm{H}), 0.97(\mathrm{~s}, 3 \mathrm{H}), 0.95-0.88(\mathrm{~m}, 2 \mathrm{H}), 0.11(\mathrm{~s}, 18 \mathrm{H}) ;{ }^{13} \mathrm{C}$ NMR $(75 \mathrm{MHz}$, benzene-d 6$) \delta 134.8$, 134.4, 129.0, 127.5, 97.9, 97.5, 88.0, 80.6, 73.9, 70.9, 70.3, 61.0, 58.8, 54.2, 53.8, 51.4, 48.3, 47.4, 47.1, 47.0, 39.3, 37.8, 36.3, 35.3, 34.2, 34.0, 33.8, 32.5, 32.4, 31.5, 20.6, 20.5, 18.7, 17.6, 0.8, 0.7; $\mathrm{MS}\left(\mathrm{ES}^{+}\right)$calcd for $\mathrm{C}_{20} \mathrm{H}_{31} \mathrm{O}_{2} \mathrm{Si}(\mathrm{M}+\mathrm{H})^{+} 331.21$, found 331.1 .

To a solution of $27 \mathrm{~s} 1$ and $28 \mathrm{~s} 1$ (100 $\mathrm{mg}, 0.30 \mathrm{mmol}, 1.0$ equiv) in methylene chloride $(5 \mathrm{~mL})$ at $23{ }^{\circ} \mathrm{C}$ was added $m$-chloroperbenzoic acid ( $104 \mathrm{mg}, 0.60 \mathrm{mmol}, 2.0$ equiv). After stirring for $1 \mathrm{~h}$, the reaction was quenched with sodium thiosulfate (10\% in saturated aqueous sodium bicarbonate, $5 \mathrm{~mL})$. The layers were separated and the organic layer was washed with saturated aqueous sodium bicarbonate $(5 \mathrm{~mL})$, brine $(5 \mathrm{~mL})$, dried over anhydrous sodium sulfate, filtered, and concentrated, to give epoxides 27s2 and 28s2 as white solid:: $R_{f}=0.18\left(10 \%\right.$ ethyl acetate-hexanes); FTIR (neat, $\mathrm{cm}^{-1}$ ) 2955, 1249, 1068, 836; ${ }^{1} \mathrm{H}$ NMR $\left(500 \mathrm{MHz}\right.$, benzene- $\left.d_{6}\right) \delta 4.32(\mathrm{dd}, J=7.7,2.4 \mathrm{~Hz}, 1 \mathrm{H}), 4.09-4.05$ $(\mathrm{m}, 1 \mathrm{H}), 3.91(\mathrm{dd}, J=8.7,2.4 \mathrm{~Hz}, 1 \mathrm{H}), 3.84(\mathrm{~d}, J=8.3 \mathrm{~Hz}, 1 \mathrm{H}), 3.60(\mathrm{~d}, J=8.7 \mathrm{~Hz}, 1 \mathrm{H}), 3.34(\mathrm{~d}, J=$ $7.8 \mathrm{~Hz}, 1 \mathrm{H}), 3.05(\mathrm{~d}, J=1.8 \mathrm{~Hz}, 1 \mathrm{H}), 2.88(\mathrm{~d}, J=5.3 \mathrm{~Hz}, 1 \mathrm{H}), 2.75(\mathrm{~m}, 2 \mathrm{H}), 2.67(\mathrm{~d}, J=1.8 \mathrm{~Hz}, 1 \mathrm{H})$, 
$2.56(\mathrm{~m}, 1 \mathrm{H}), 2.25(\mathrm{ddd}, J=11.8,9.2,2.4 \mathrm{~Hz}, 1 \mathrm{H}), 2.17-2.13(\mathrm{~m}, 2 \mathrm{H}), 2.08-2.06(\mathrm{~m}, 1 \mathrm{H}), 2.00-1.92$ $(\mathrm{m}, 3 \mathrm{H}), 1.89-1.83(\mathrm{~m}, 1 \mathrm{H}), 1.68-1.49(\mathrm{~m}, 9 \mathrm{H}), 1.33-1.46(\mathrm{~m}, 4 \mathrm{H}), 1.21-1.17(\mathrm{dd}, J=14.0,6.5 \mathrm{~Hz}$, $1 \mathrm{H}), 0.99(\mathrm{~s}, 3 \mathrm{H}), 0.94(\mathrm{~s}, 3 \mathrm{H}), 0.89-0.79(\mathrm{~m}, 2 \mathrm{H}), 0.12(\mathrm{~s}, 18 \mathrm{H}) ;{ }^{13} \mathrm{C}$ NMR $\left(75 \mathrm{MHz}\right.$, benzene- $\left.d_{6}\right) \delta$ 90.1, 89.7, 89.3, 81.2, 73.6, 70.2, 65.3, 60.8, 58.7, 57.8, 57.7, 56.8, 53.9, 53.4, 49.7, 48.2, 47.6, 46.9, 46.1, 44.6, 38.9, 38.8, 36.3, 36.1, 35.1, 33.4, 33.0, 32.9, 32.4, 29.4, 20.6, 20.4, 18.7, 17.6, 0.8, 0.6; $\mathrm{MS}\left(\mathrm{ES}^{+}\right.$) calcd for $\mathrm{C}_{20} \mathrm{H}_{31} \mathrm{O}_{3} \mathrm{Si}(\mathrm{M}+\mathrm{H})^{+} 347.20$, found 347.1 .

To a solution of epoxides 27s 2 and $28 \mathrm{~s} 2$ (104 $\mathrm{mg}, 0.30 \mathrm{mmol}, 1.0$ equiv) in methol was added $p$ toluenesulfonic acid ( $11.5 \mathrm{mg}, 0.60 \mathrm{mmol}, 2.0$ equiv) at $23{ }^{\circ} \mathrm{C}$. After stirring for $5 \mathrm{~min}$, the reaction was quenched with saturated aqueous sodium bicarbonate $(5 \mathrm{~mL})$, The mixture was extracted with ethyl acetate $(3 \times 10 \mathrm{~mL})$ and the combined organic was washed with saturated aqueous sodium bicarbonate $(5 \mathrm{~mL})$, brine $(5 \mathrm{~mL})$, dried over anhydrous sodium sulfate, filtered, concentrated, and purified by silica gel column chromatography to give 27 (29 mg, 30\% over three steps) and $\mathbf{2 8}$ (33 mg, $35 \%$ over three steps) as white solid.

27: $R_{f}=0.29\left(50 \%\right.$ ethyl acetate-hexanes); FTIR (neat, $\left.\mathrm{cm}^{-1}\right) 3442,2949,2879,1049,915 ;{ }^{1} \mathrm{H}$ NMR $\left(500 \mathrm{MHz}\right.$, benzene- $\left.d_{6}\right) \delta 5.80(\mathrm{dd}, J=9.5,4.0 \mathrm{~Hz}, 1 \mathrm{H}), 5.46(\mathrm{dd}, J=9.5,6.5 \mathrm{~Hz}, 1 \mathrm{H}), 4.10(\mathrm{br}, 1 \mathrm{H})$, $3.92(\mathrm{dd}, J=9.0,1.7 \mathrm{~Hz}, 1 \mathrm{H}), 3.74-3.65(\mathrm{~m}, 2 \mathrm{H}), 3.43$ (d, $J=9.0 \mathrm{~Hz}, 1 \mathrm{H}), 2.95-2.85$ (m, 1H), 2.88 (s, $3 \mathrm{H}), 2.48(\mathrm{dd}, J=6.3,5.0 \mathrm{~Hz}, 1 \mathrm{H}), 1.87(\mathrm{dd}, J=13.2,8.4 \mathrm{~Hz}, 1 \mathrm{H}), 1.75-1.25(\mathrm{~m}, 7 \mathrm{H}), 1.12$ (ddd, $J=$ 16.6, 11.4, 5.1, $1 \mathrm{H}), 0.87(\mathrm{~s}, 3 \mathrm{H}) ;{ }^{13} \mathrm{C}$ NMR $\left(75 \mathrm{MHz}\right.$, benzene- $\left.d_{6}\right) \delta 132.1,127.5,117.4,78.9,78.4$, $71.0,58.4,55.2,50.0,48.2,38.9,38.2,37.7,36.4,35.5,33.9,20.1,17.5 ; \mathrm{MS}(\mathrm{EI})$ calcd for $\mathrm{C}_{18} \mathrm{H}_{26} \mathrm{O}_{4}$ $(\mathrm{M})^{+} 306$, found 306 .

28: $R_{f}=0.23\left(50 \%\right.$ ethyl acetate-hexanes); FTIR (neat, $\left.\mathrm{cm}^{-1}\right) 3397,2942,2880,1006 ;{ }^{1} \mathrm{H}$ NMR $(500$ MHz, benzene- $\left.d_{6}\right) \delta 5.93(\mathrm{~d}, J=9.6 \mathrm{~Hz}, 1 \mathrm{H}), 5.69(\mathrm{ddd}, J=9.6,3.7,1.3 \mathrm{~Hz}, 1 \mathrm{H}), 3.95(\mathrm{dt}, J=11.6$, $2.8 \mathrm{~Hz}, 1 \mathrm{H}), 3.69(\mathrm{t}, J=8.9 \mathrm{~Hz}, 2 \mathrm{H}), 3.32(\mathrm{~d}, J=9.3 \mathrm{~Hz}, 1 \mathrm{H}), 3.07$ (s, 3H), 2.69 (dddd, $J=6.7,2.1$, $1.2,1.2 \mathrm{~Hz}, 1 \mathrm{H}), 2.61(\mathrm{~d}, J=11.7 \mathrm{~Hz}, 1 \mathrm{H}), 2.07-1.21(\mathrm{~m}, 9 \mathrm{H}), 1.04-0.92(\mathrm{~m}, 1 \mathrm{H}), 0.82(\mathrm{~s}, 3 \mathrm{H}) ;{ }^{13} \mathrm{C}$ NMR $\left(75 \mathrm{MHz}\right.$, benzene- $\left.d_{6}\right) \delta 135.1,128.0,115.7,79.1,76.0,73.5,56.5,54.8,49.7,49.1,43.0,42.9$, $42.7,39.1,33.8,33.3,20.4,19.2$.

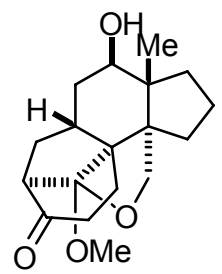

$\left(1 S^{*}, 5 R^{*}, 6 R^{*}, 8 R^{*}, 10 R^{*}, 14 R^{*}, 17 R^{*}\right)-6-H y d r o x y l-17-m e t h o x y l-5-m e t h y l-11-0 x 0-16-$ oxapentacyclo[7.4.3.0 $\left.{ }^{1,5} \cdot 0^{8,14} \cdot 0^{14,17}\right]$ heptadecane (29). To a solution of 28 ( $10.0 \mathrm{mg}$, $0.03 \mathrm{mmol}, 1$ equiv) in methol was add palladium on carbon $(30 \% \mathrm{w} / \mathrm{w}, 1 \mathrm{mg})$ was added and the solution was purged with hydrogen for $5 \mathrm{~min}$. After stirring for an additional $30 \mathrm{~min}$, the reaction was filted through a pad of Celite and concentrated to give 29 as white solid $(9.4 \mathrm{mg}, 94 \%)$. Recrystallization from diethyl ether gave colorless prisms suitable for single X-ray crystallographic analysis (CCDC 659036): $R_{f}=0.25$ (50\% ethyl acetate-hexanes); $R_{f}=0.25$ (50\% ethyl acetate-hexanes); FTIR (neat, $\mathrm{cm}^{-1}$ ) 3441, 2958, 1711, 1455 , 1057; ${ }^{1} \mathrm{H}$ NMR $\left(500 \mathrm{MHz}\right.$, benzene- $\left.d_{6}\right) \delta 3.78(\mathrm{~d}, J=9.1 \mathrm{~Hz}, 1 \mathrm{H}), 3.73(\mathrm{~m}, 1 \mathrm{H}), 3.34(\mathrm{~d}, J=9.2 \mathrm{~Hz}$, $1 \mathrm{H}), 3.18(\mathrm{~s}, 3 \mathrm{H}), 3.16(\mathrm{~d}, J=7.6 \mathrm{~Hz}, 1 \mathrm{H}), 2.16-2.12(\mathrm{~m}, 1 \mathrm{H}), 2.02-1.97(\mathrm{~m}, 2 \mathrm{H}), 1.87-1.84(\mathrm{~m}, 1 \mathrm{H})$, 1.75 (ddd, $J=13.3,7.2,7.2 \mathrm{~Hz}, 2 \mathrm{H}), 1.63-1.49$ (m, $5 \mathrm{H}), 1.41-1.36(\mathrm{~m}, 1 \mathrm{H}), 1.32-1.27$ (m, $1 \mathrm{H}), 1.17-$ $1.14(\mathrm{~m}, 1 \mathrm{H}), 1.02-1.00(\mathrm{~m}, 1 \mathrm{H}), 0.74(\mathrm{~s}, 3 \mathrm{H}) ;{ }^{13} \mathrm{C}$ NMR $\left(75 \mathrm{MHz}\right.$, benzene- $\left.d_{6}\right) \delta 209.0,116.9,79.0$,

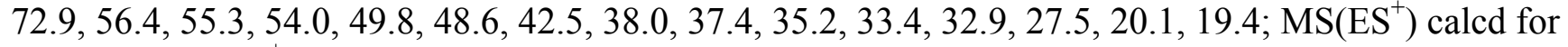
$\mathrm{C}_{18} \mathrm{H}_{27} \mathrm{O}_{4}(\mathrm{M}+\mathrm{H})^{+} 307.19$, found 307.21 . 

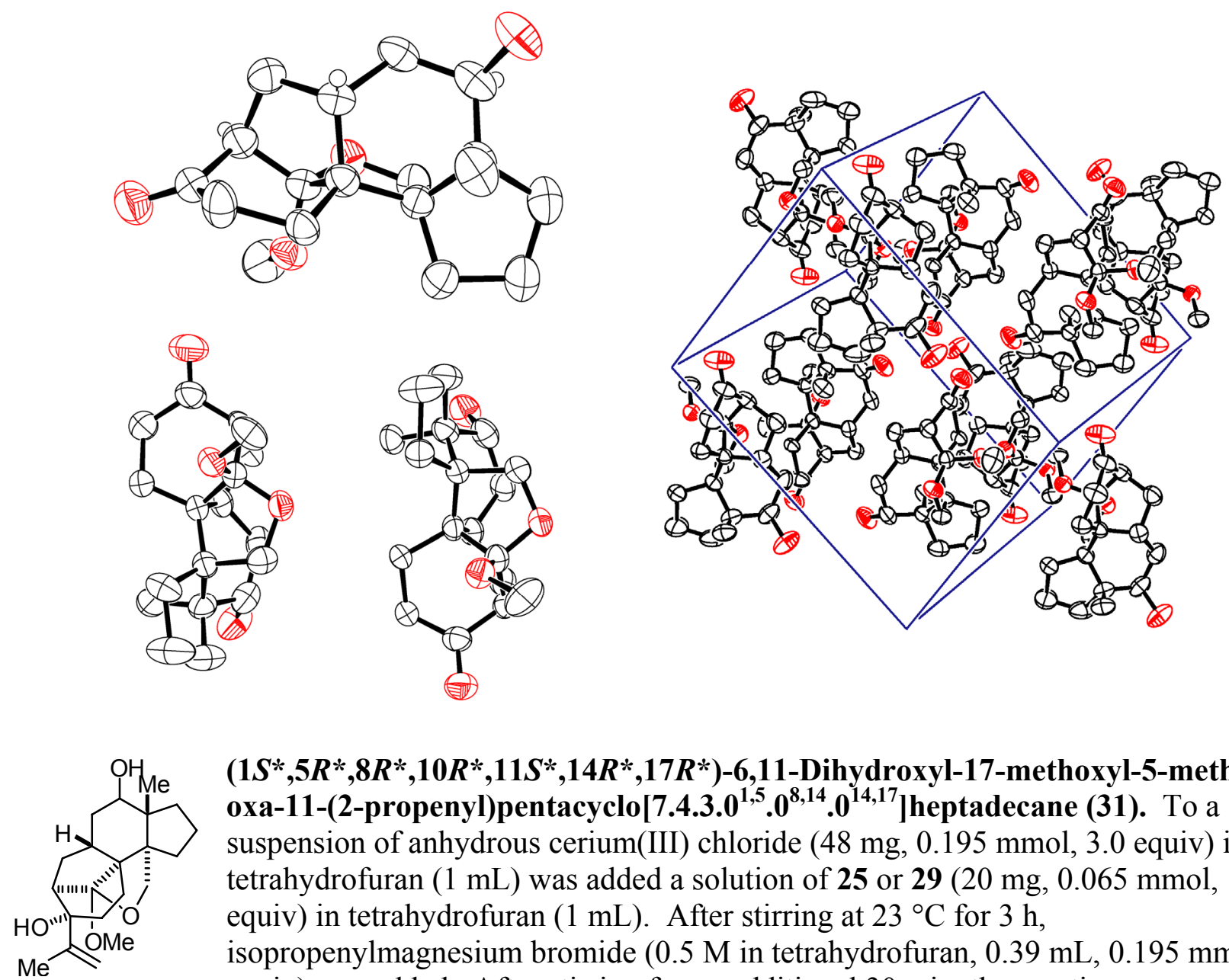

$\left(1 S^{*}, 5 R^{*}, 8 R^{*}, 10 R^{*}, 11 S^{*}, 14 R^{*}, 17 R^{*}\right)-6,11-D i h y d r o x y l-17-m e t h o x y l-5-m e t h y l-16-$ oxa-11-(2-propenyl)pentacyclo $\left[7.4 .3 .0^{1,5} \cdot 0^{8,14} \cdot 0^{14,17}\right]$ heptadecane (31). To a suspension of anhydrous cerium(III) chloride ( $48 \mathrm{mg}, 0.195 \mathrm{mmol}, 3.0$ equiv) in tetrahydrofuran $(1 \mathrm{~mL})$ was added a solution of 25 or $29(20 \mathrm{mg}, 0.065 \mathrm{mmol}, 1.0$ equiv) in tetrahydrofuran $(1 \mathrm{~mL})$. After stirring at $23{ }^{\circ} \mathrm{C}$ for $3 \mathrm{~h}$, isopropenylmagnesium bromide $(0.5 \mathrm{M}$ in tetrahydrofuran, $0.39 \mathrm{~mL}, 0.195 \mathrm{mmol}, 3.0$ equiv) was added. After stirring for an additional $30 \mathrm{~min}$, the reaction was quenched with water $(2 \mathrm{~mL})$. The mixture was extracted with diethyl ether $(10 \mathrm{~mL} \times 3)$ and the combined organic was washed with saturated aqueous sodium bicarbonate $(5 \mathrm{~mL})$, brine $(5 \mathrm{~mL})$, dried over anhydrous sodium sulfate, filtered, concentrated, and purified by silica gel column chromatography to give $\mathbf{3 1}$ as white solid (20 mg, 90\%). Recrystallization of 31a from diethyl ether gave colorless prisms suitable for single X-ray crystallographic analysis (CCDC 659038).

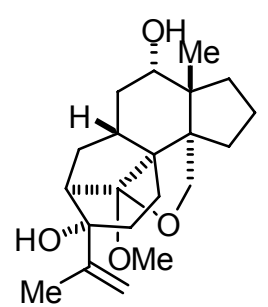

31a: $R_{f}=0.50$ (50\% ethyl acetate-hexanes); FTIR (neat, $\left.\mathrm{cm}^{-1}\right) 3456,2960,2879$, 1458, 1100, 902; ${ }^{1} \mathrm{H}$ NMR $\left(500 \mathrm{MHz}\right.$, benzene- $\left.d_{6}\right) \delta 5.10(\mathrm{~s}, 1 \mathrm{H}), 5.00(\mathrm{~s}, 1 \mathrm{H}), 4.98(\mathrm{~s}$, $1 \mathrm{H}), 4.51(\mathrm{~d}, J=8.8 \mathrm{~Hz}, 1 \mathrm{H}), 3.36(\mathrm{~d}, J=8.9 \mathrm{~Hz}, 1 \mathrm{H}), 3.34(\mathrm{t}, J=8.5 \mathrm{~Hz}, 1 \mathrm{H}), 3.13$ $(\mathrm{s}, 3 \mathrm{H}), 2.54(\mathrm{~d}, J=6.8 \mathrm{~Hz}, 1 \mathrm{H}), 2.09-2.03(\mathrm{~m}, 1 \mathrm{H}), 2.00(\mathrm{dd}, J=13.1,7.9 \mathrm{~Hz}, 1 \mathrm{H})$, $1.97(\mathrm{~s}, 3 \mathrm{H}), 1.83-1.77(\mathrm{~m}, 2 \mathrm{H}), 1.71(\mathrm{ddd}, J=17.4,8.8,8.8 \mathrm{~Hz}, 1 \mathrm{H}), 1.65-1.54$ (m, $4 \mathrm{H}), 1.52-1.44(\mathrm{~m}, 2 \mathrm{H}), 1.24-1.20(\mathrm{~m}, 2 \mathrm{H}), 1.03-0.91(\mathrm{~m}, 2 \mathrm{H}), 0.58(\mathrm{~s}, 3 \mathrm{H}) ;{ }^{13} \mathrm{C}$ NMR $\left(75 \mathrm{MHz}\right.$, benzene- $\left.d_{6}\right) \delta 149.5,116.7,111.8,79.8,78.5,73.1,56.1,53.3,50.0$, 48.5, 42.2, 41.5, 36.3, 33.1, 33.0, 32.1, 29.9, 27.6, 26.1, 20.1, 19.3; $\mathrm{MS}\left(\mathrm{ES}^{+}\right)$calcd for $\mathrm{C}_{20} \mathrm{H}_{29} \mathrm{O}_{3}(\mathrm{M}-$ $\mathrm{OMe}^{+}$317.2117, found 317.2110.

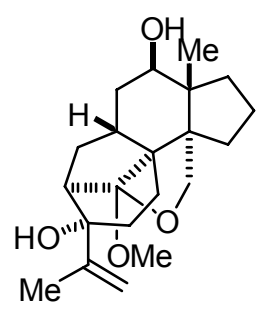

31b: $R_{f}=0.50$ (50\% ethyl acetate-hexanes); FTIR (neat, $\left.\mathrm{cm}^{-1}\right)$ 3452, 2961, 2880, 1459, 1101, 958, 736; ${ }^{1} \mathrm{H}$ NMR (500 MHz, benzene- $\left.d_{6}\right) \delta 4.98(\mathrm{~s}, 1 \mathrm{H}), 4.97(\mathrm{~s}, 1 \mathrm{H})$, $4.96(\mathrm{~s}, 1 \mathrm{H}), 3.74(\mathrm{~d}, J=6.5 \mathrm{~Hz}, 1 \mathrm{H}), 3.69(\mathrm{~d}, J=9.2 \mathrm{~Hz}, 1 \mathrm{H}), 3.19(\mathrm{~d}, J=9.2 \mathrm{~Hz}$, $1 \mathrm{H}), 3.11(\mathrm{~s}, 3 \mathrm{H}), 2.51(\mathrm{~d}, J=6.8 \mathrm{~Hz}, 1 \mathrm{H}), 2.00(\mathrm{ddd}, J=13.3,13.2,5.3 \mathrm{~Hz}, 1 \mathrm{H}), 1.96$ (s, 3H), 1.92-1.86 (m, 1H), 1.83-1.74 (m, 3H), 1.68-1.53 (m, 6H), 1.49-1.41 (m, 1H), 
$1.31(\mathrm{dd}, J=13.1,4.7 \mathrm{~Hz}, 1 \mathrm{H}), 1.11(\mathrm{ddd}, J=13.2,6.8,4.1 \mathrm{~Hz}, 1 \mathrm{H}), 1.05-1.00(\mathrm{~m}, 1 \mathrm{H}), 0.82(\mathrm{~s}, 3 \mathrm{H})$; ${ }^{13} \mathrm{C}$ NMR $\left(75 \mathrm{MHz}\right.$, benzene- $\left.d_{6}\right) \delta 149.3,116.5,112.0,78.4,78.3,73.6,56.4,53.4,50.1,49.2,43.4$, $41.7,39.2,35.3,33.9,32.7,29.8,26.4,20.3,19.9,19.3$; $M S\left(E^{+}\right)$calcd for $\mathrm{C}_{20} \mathrm{H}_{29} \mathrm{O}_{3}(\mathrm{M}-\mathrm{OMe})^{+}$
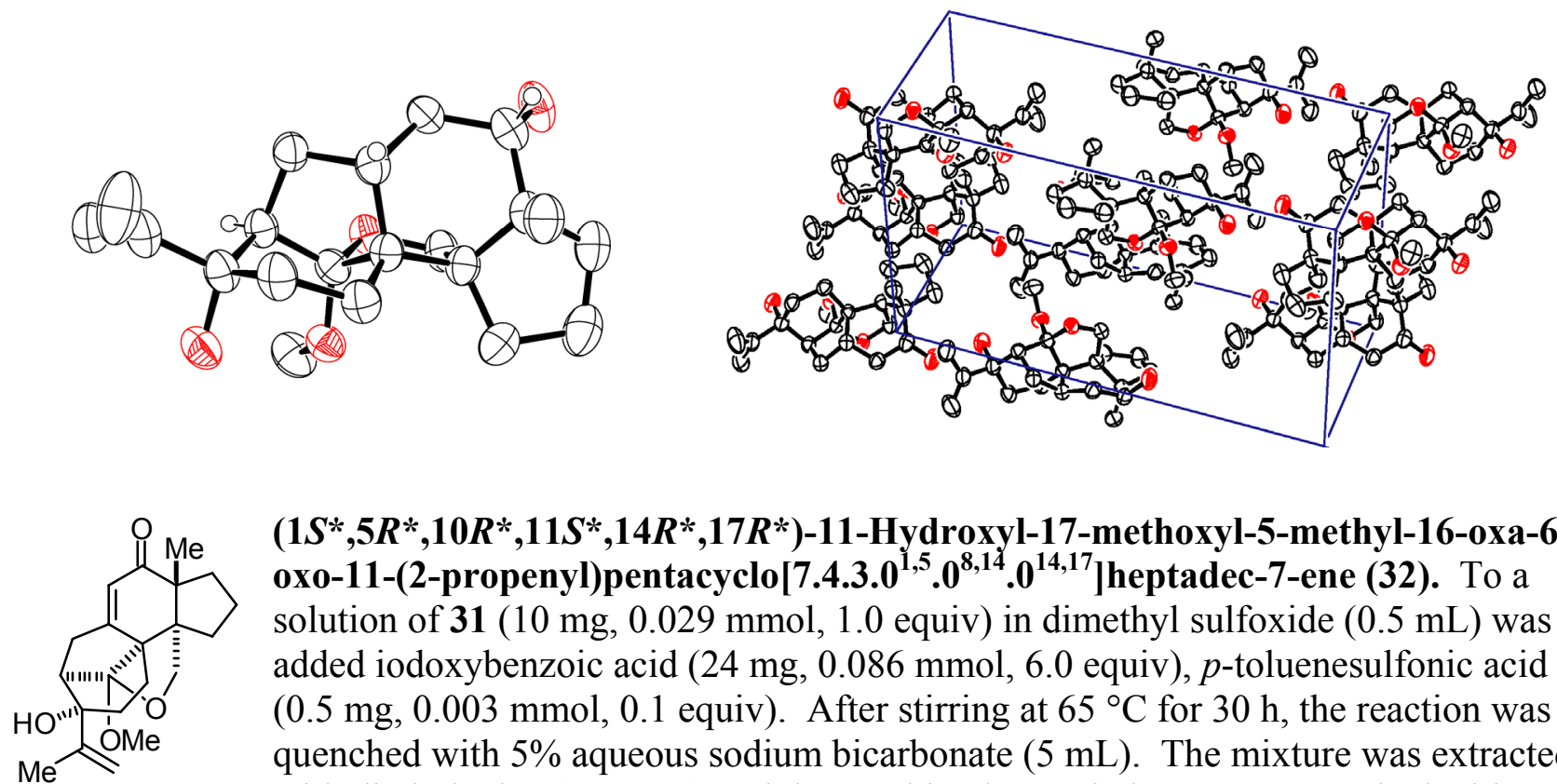

$\left(1 S^{*}, 5 R^{*}, 10 R^{*}, 11 S^{*}, 14 R^{*}, 17 R^{*}\right)-11-H y d r o x y l-17-m e t h o x y l-5-m e t h y l-16-0 x a-6-$ oxo-11-(2-propenyl)pentacyclo $\left[7.4 .3 .0^{1,5} \cdot 0^{8,14} \cdot 0^{14,17}\right]$ heptadec-7-ene (32). To a solution of 31 (10 mg, $0.029 \mathrm{mmol}, 1.0$ equiv) in dimethyl sulfoxide $(0.5 \mathrm{~mL})$ was added iodoxybenzoic acid ( $24 \mathrm{mg}, 0.086 \mathrm{mmol}, 6.0$ equiv), $p$-toluenesulfonic acid ( $0.5 \mathrm{mg}, 0.003 \mathrm{mmol}, 0.1$ equiv). After stirring at $65^{\circ} \mathrm{C}$ for $30 \mathrm{~h}$, the reaction was quenched with $5 \%$ aqueous sodium bicarbonate $(5 \mathrm{~mL})$. The mixture was extracted with diethyl ether $(3 \times 5 \mathrm{~mL})$ and the combined organic layers were washed with saturated aqueous sodium bicarbonate $(5 \mathrm{~mL})$, brine $(5 \mathrm{~mL})$, dried over anhydrous sodium sulfate, filtered, concentrated, and purified by silica gel column chromatography to give 32 as colorless oil ( 6.0 $\mathrm{mg}, 60 \%$ ): $R_{f}=0.33$ (30\% ethyl acetate-hexanes); FTIR (neat, $\mathrm{cm}^{-1}$ ) 3475, 2964, 1673, 1133, 1057; ${ }^{1} \mathrm{H}$ NMR $\left(500 \mathrm{MHz}\right.$, benzene- $\left.d_{6}\right) \delta 5.85(\mathrm{~s}, 1 \mathrm{H}), 4.90(\mathrm{~s}, 1 \mathrm{H}), 4.85(\mathrm{~s}, 1 \mathrm{H}), 4.80(\mathrm{~s}, 1 \mathrm{H}), 3.52(\mathrm{dd}, J=8.8$, $1.4 \mathrm{~Hz}, 1 \mathrm{H}), 3.30$ (d, $J=8.8 \mathrm{~Hz}, 1 \mathrm{H}), 3.05$ (s, 3H), 2.56 (d, $J=6.6 \mathrm{~Hz}, 1 \mathrm{H}), 2.05-2.16$ (m, 2H), 1.95$1.91(\mathrm{~m}, 1 \mathrm{H}), 1.89(\mathrm{~s}, 3 \mathrm{H}), 1.46-1.72(\mathrm{~m}, 8 \mathrm{H}), 1.29-1.36(\mathrm{~m}, 1 \mathrm{H}), 0.99(\mathrm{~s}, 3 \mathrm{H}) ;{ }^{13} \mathrm{C} \mathrm{NMR}(75 \mathrm{MHz}$, benzene- $\left.d_{6}\right) \delta 201.0,166.5,148.6,127.0,115.0,112.2,81.0,78.3,57.6,57.0,53.7,50.5,41.5,33.6$, 32.7, 30.1, 29.4, 23.9, 23.1, 19.1, 19.0; $\mathrm{MS}\left(\mathrm{ES}^{+}\right)$calcd for $\mathrm{C}_{21} \mathrm{H}_{29} \mathrm{O}_{4}(\mathrm{M}+\mathrm{H})^{+}$345.21, found 345.27. 


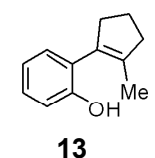

13
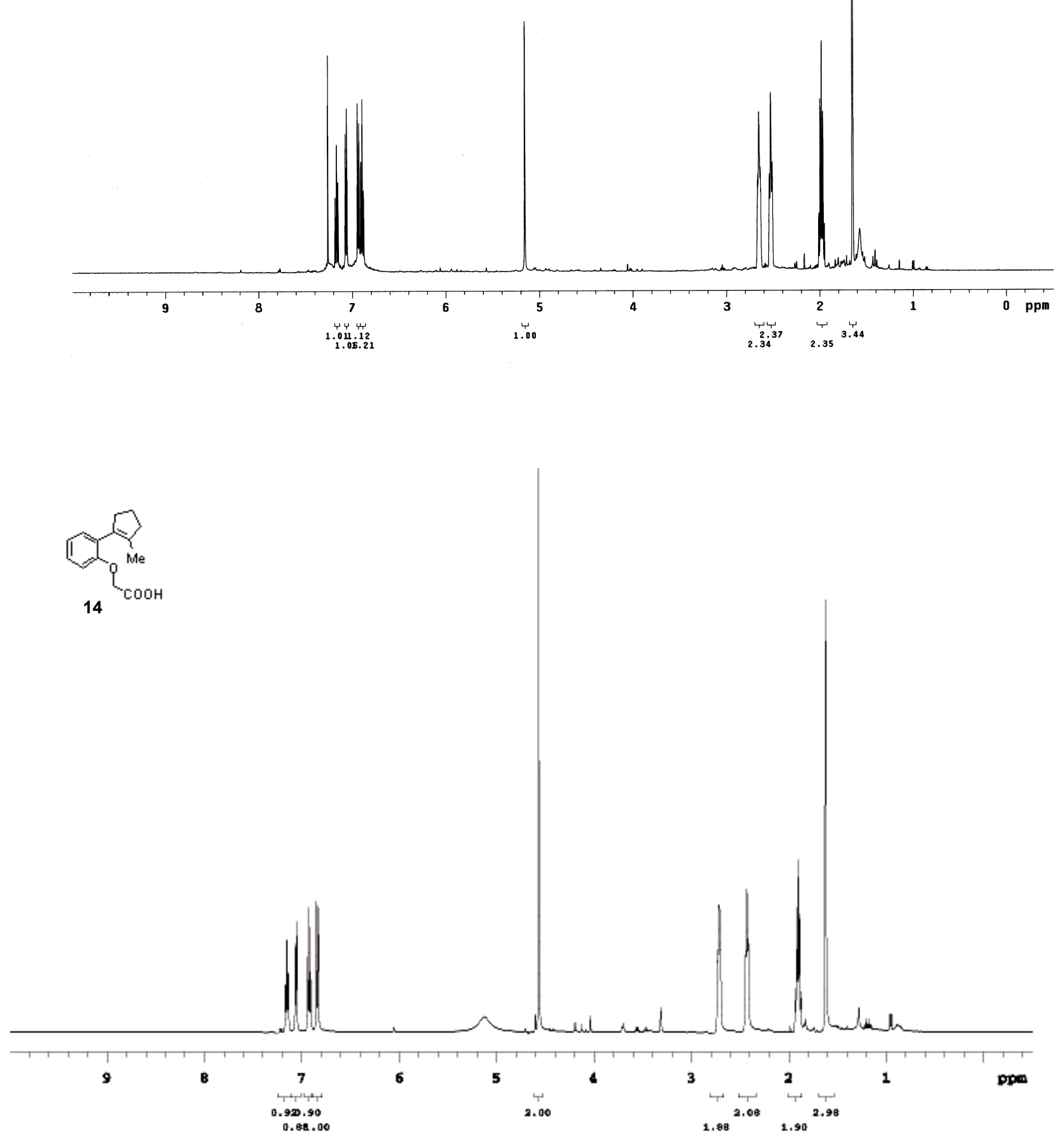


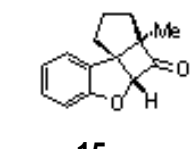

15
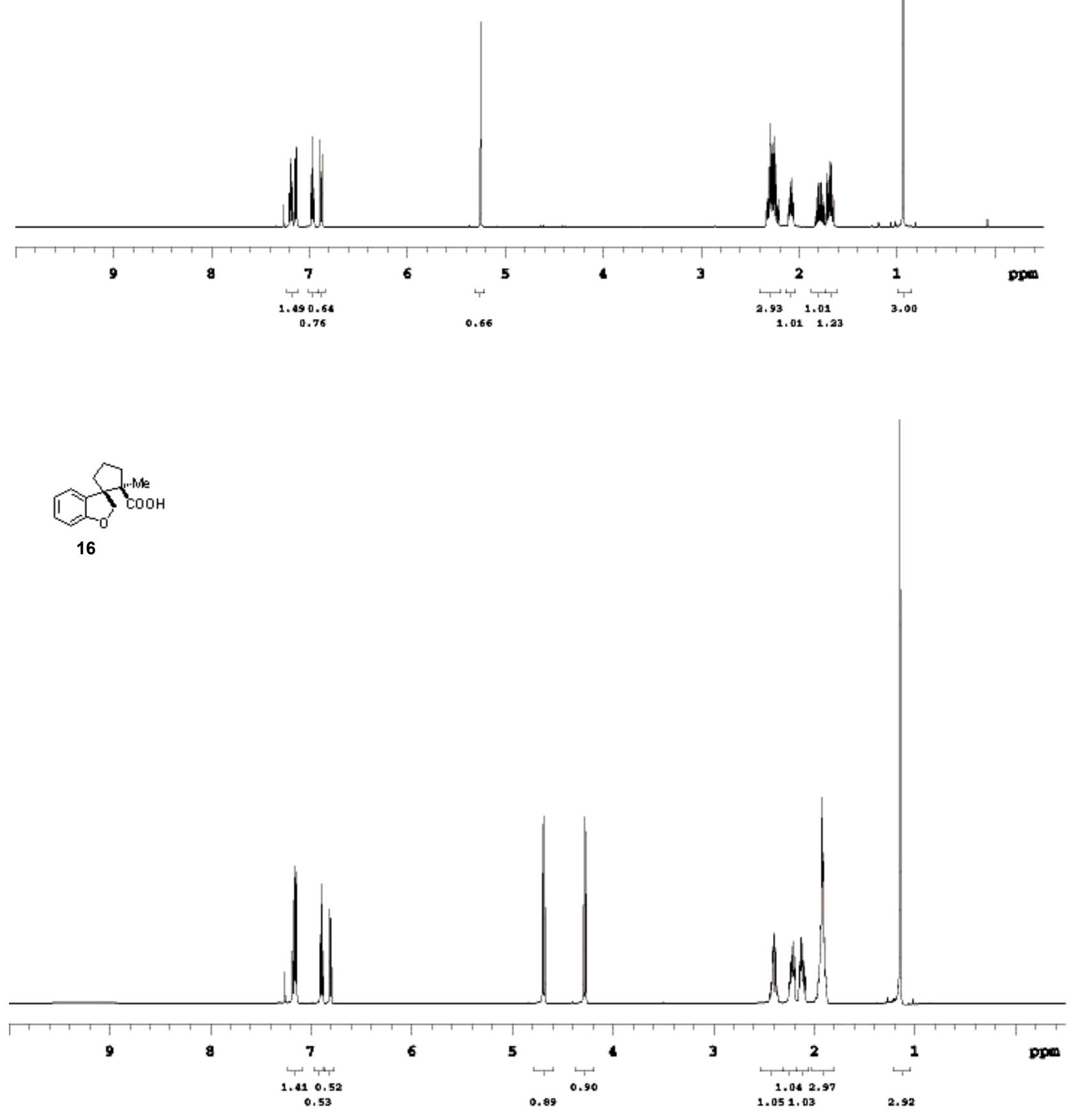

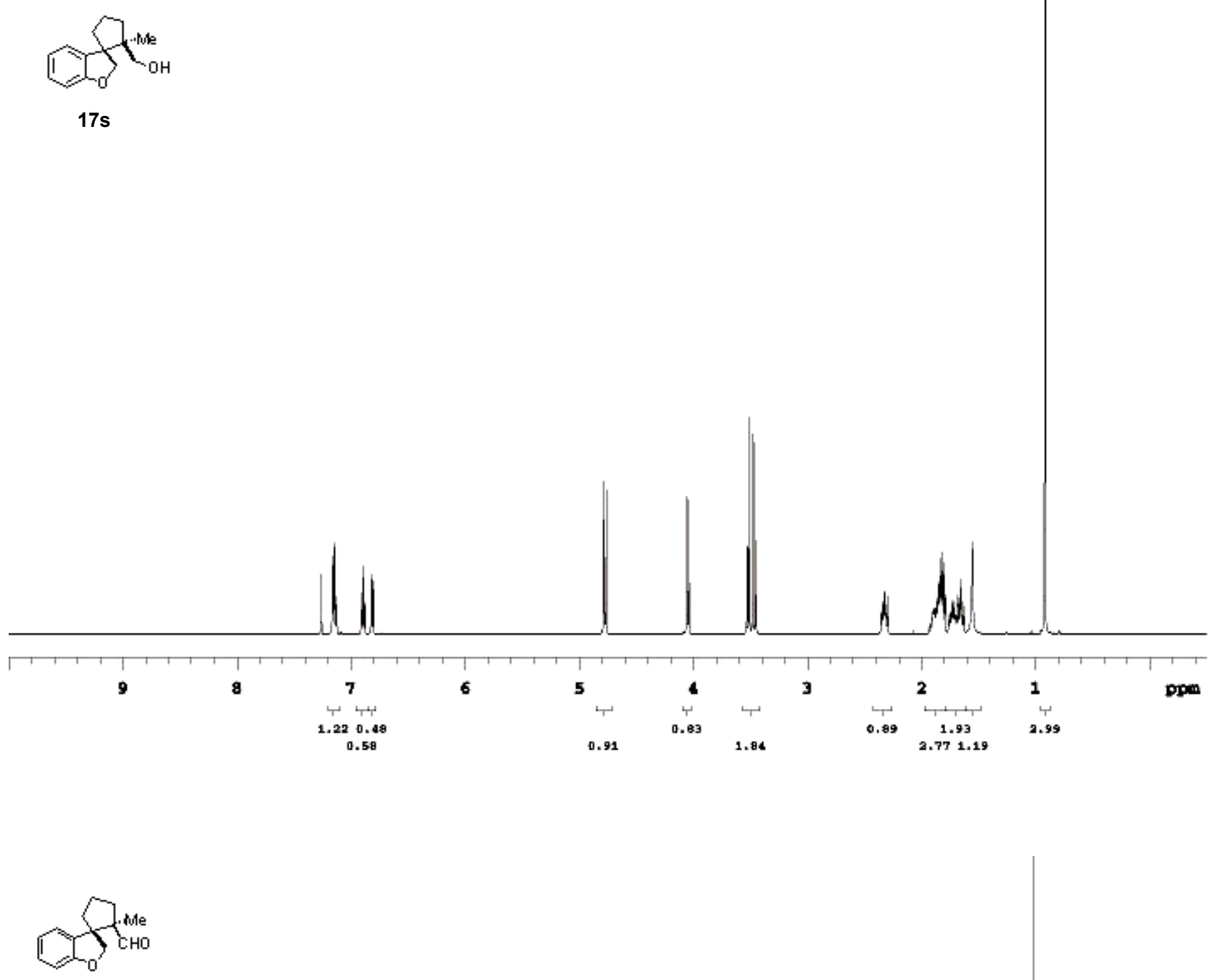

17

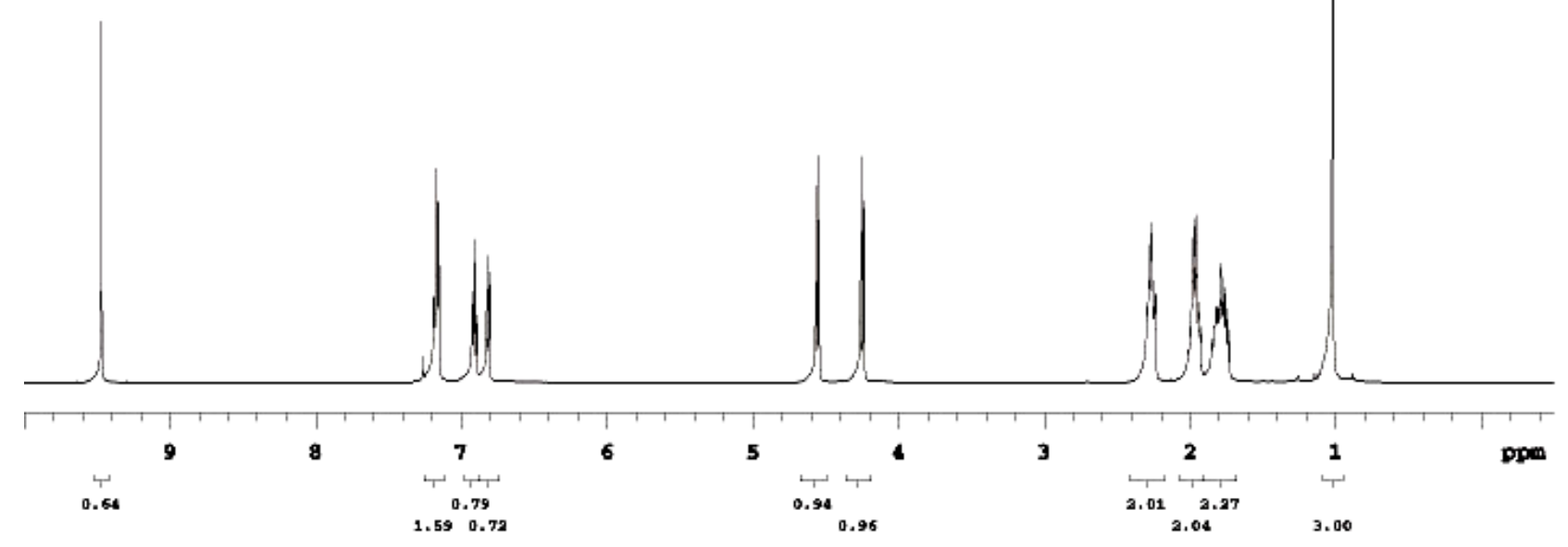



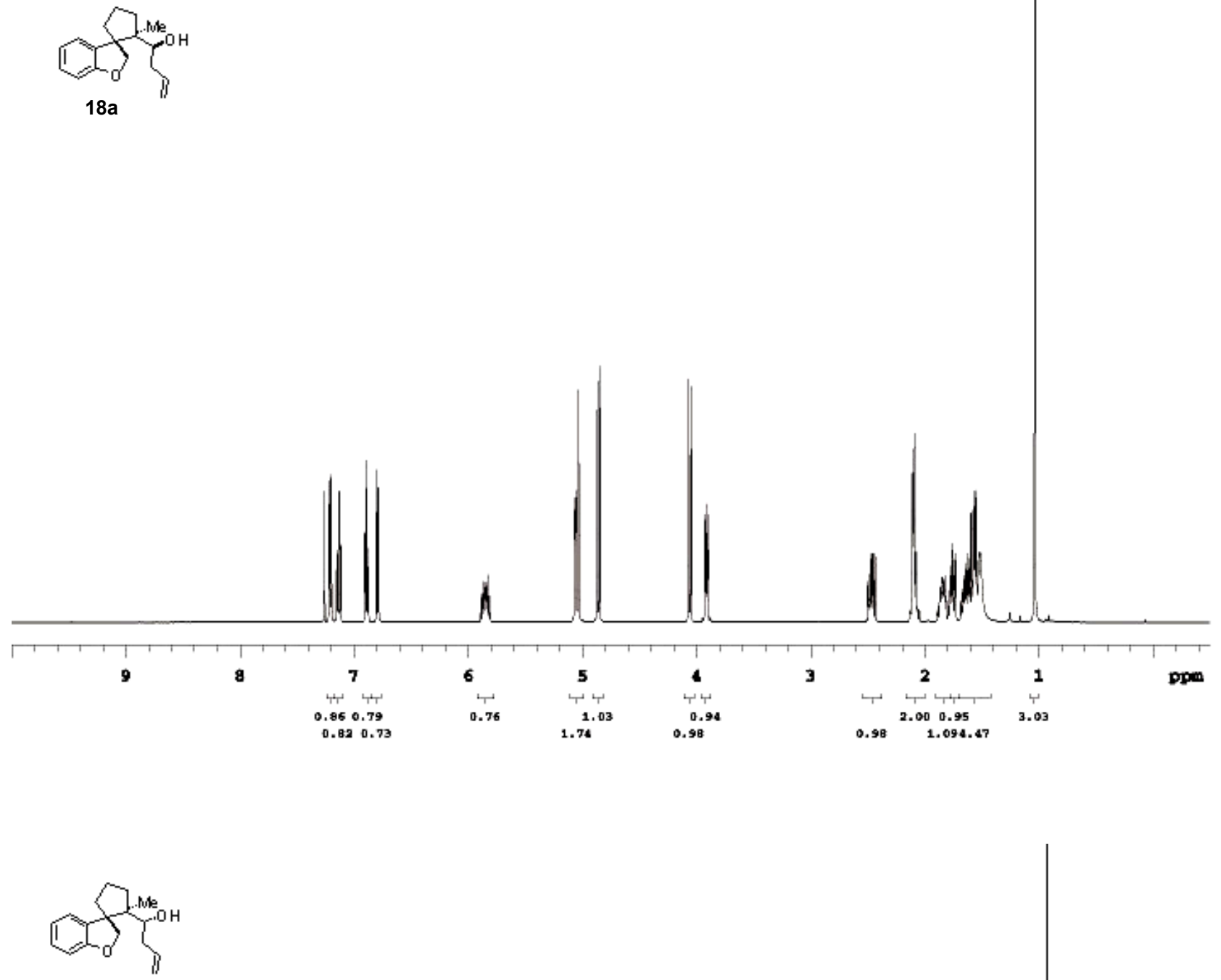

$18 b$

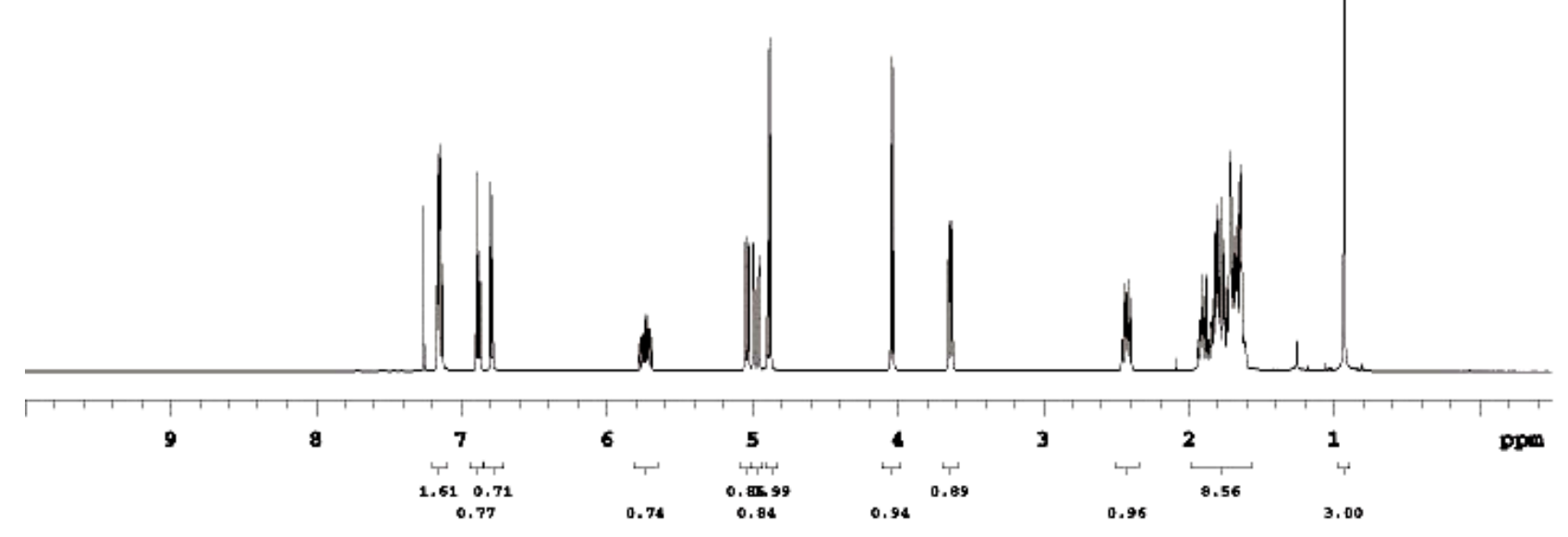




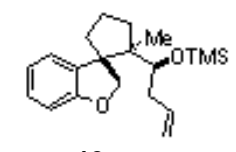

19

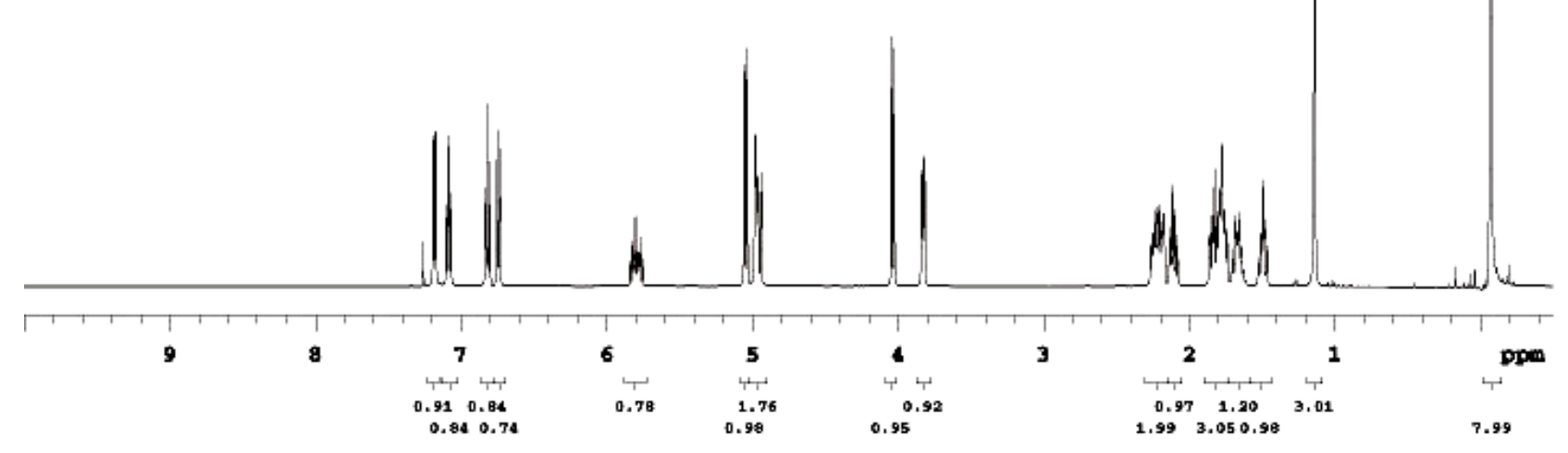

$c_{0}$

20

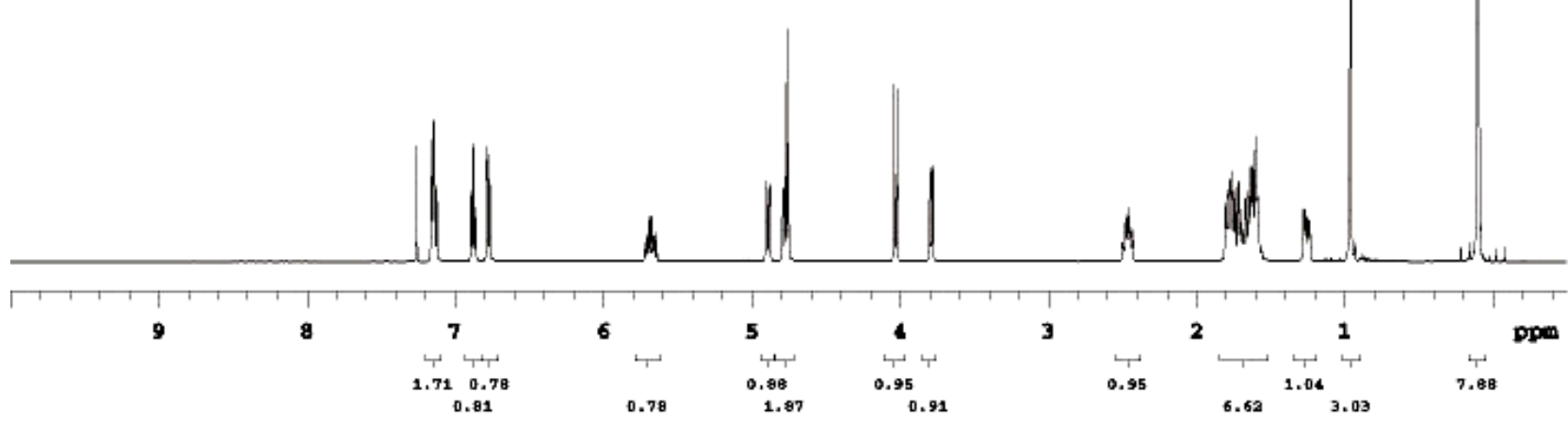



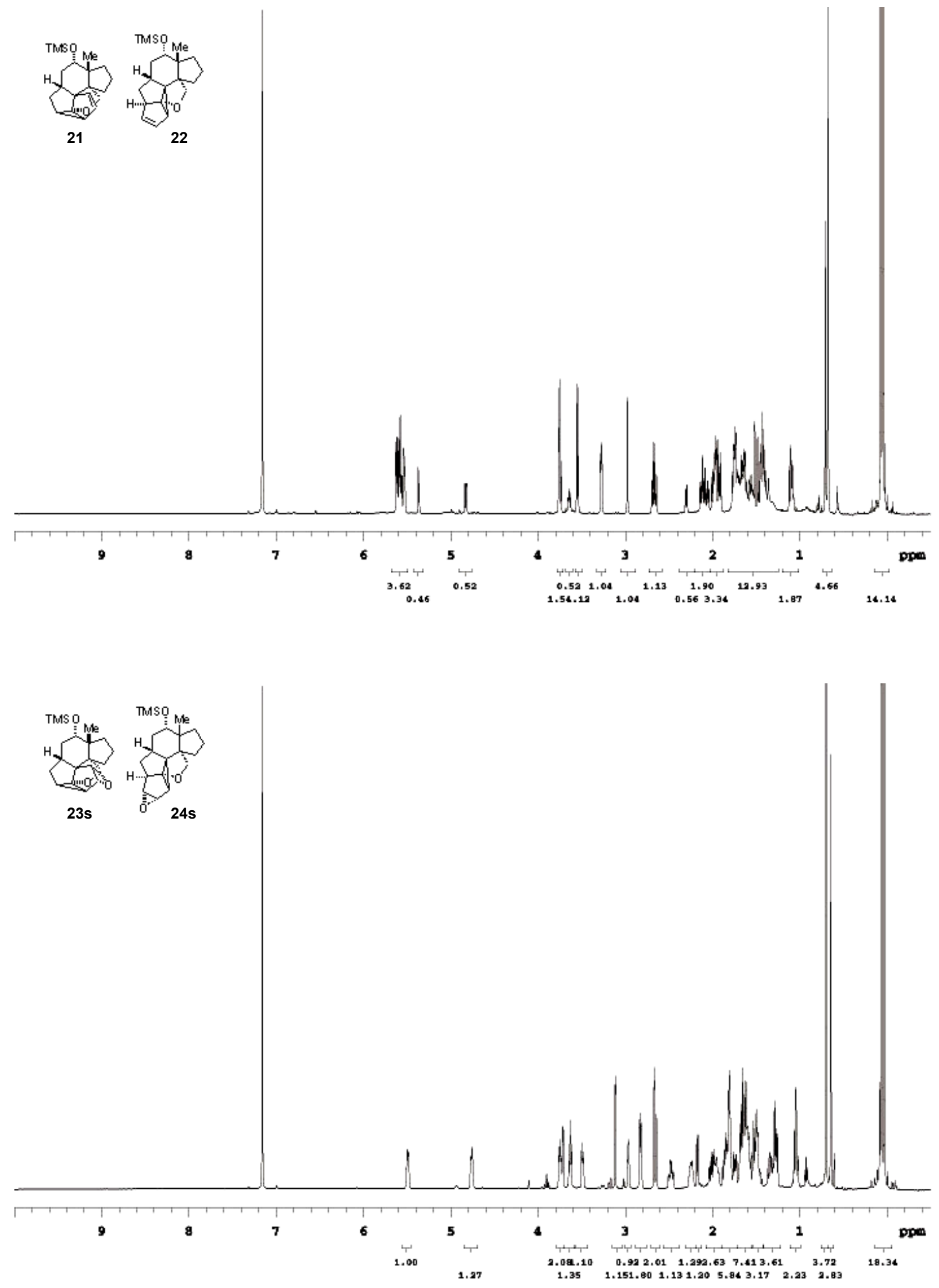

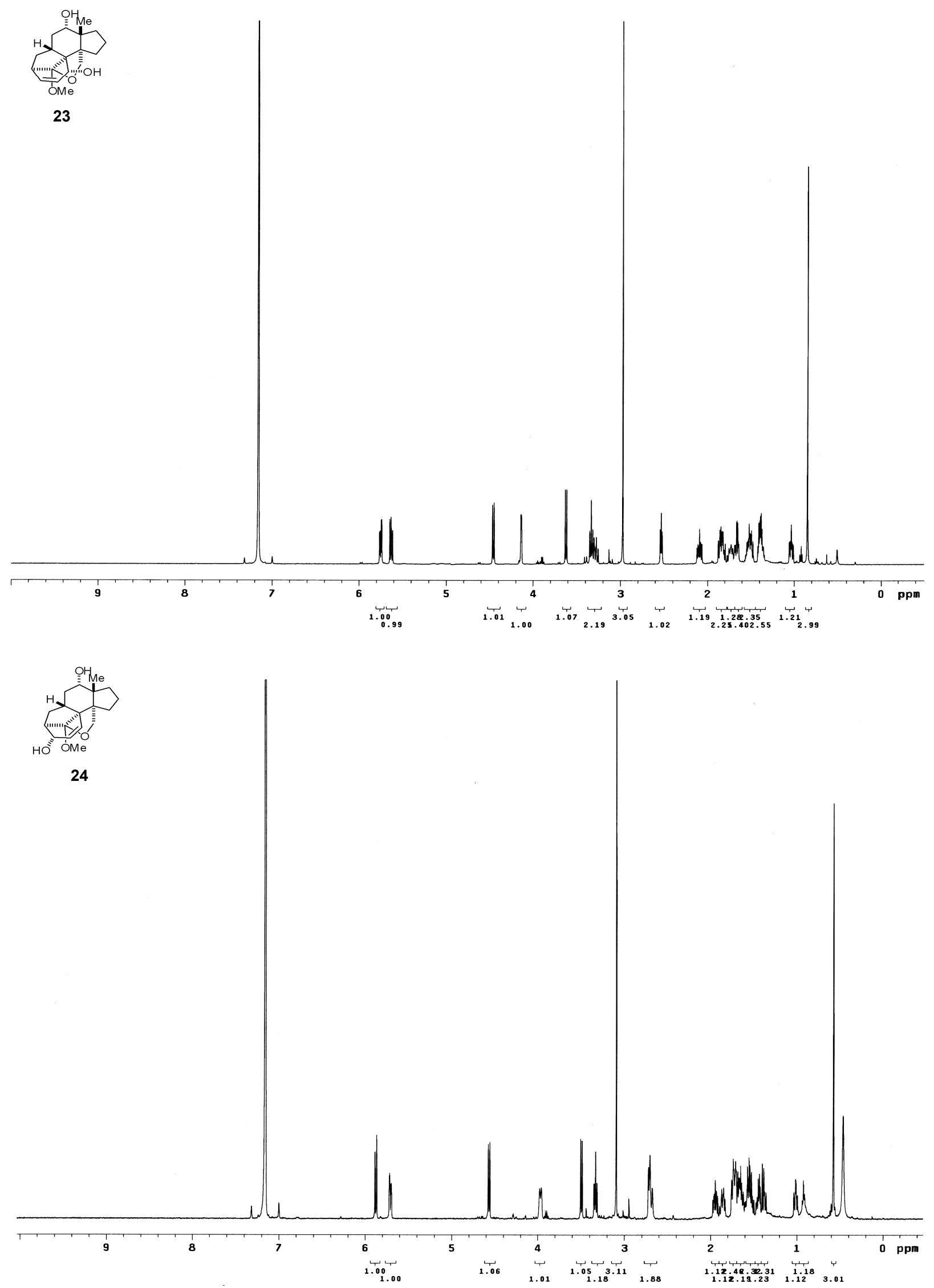

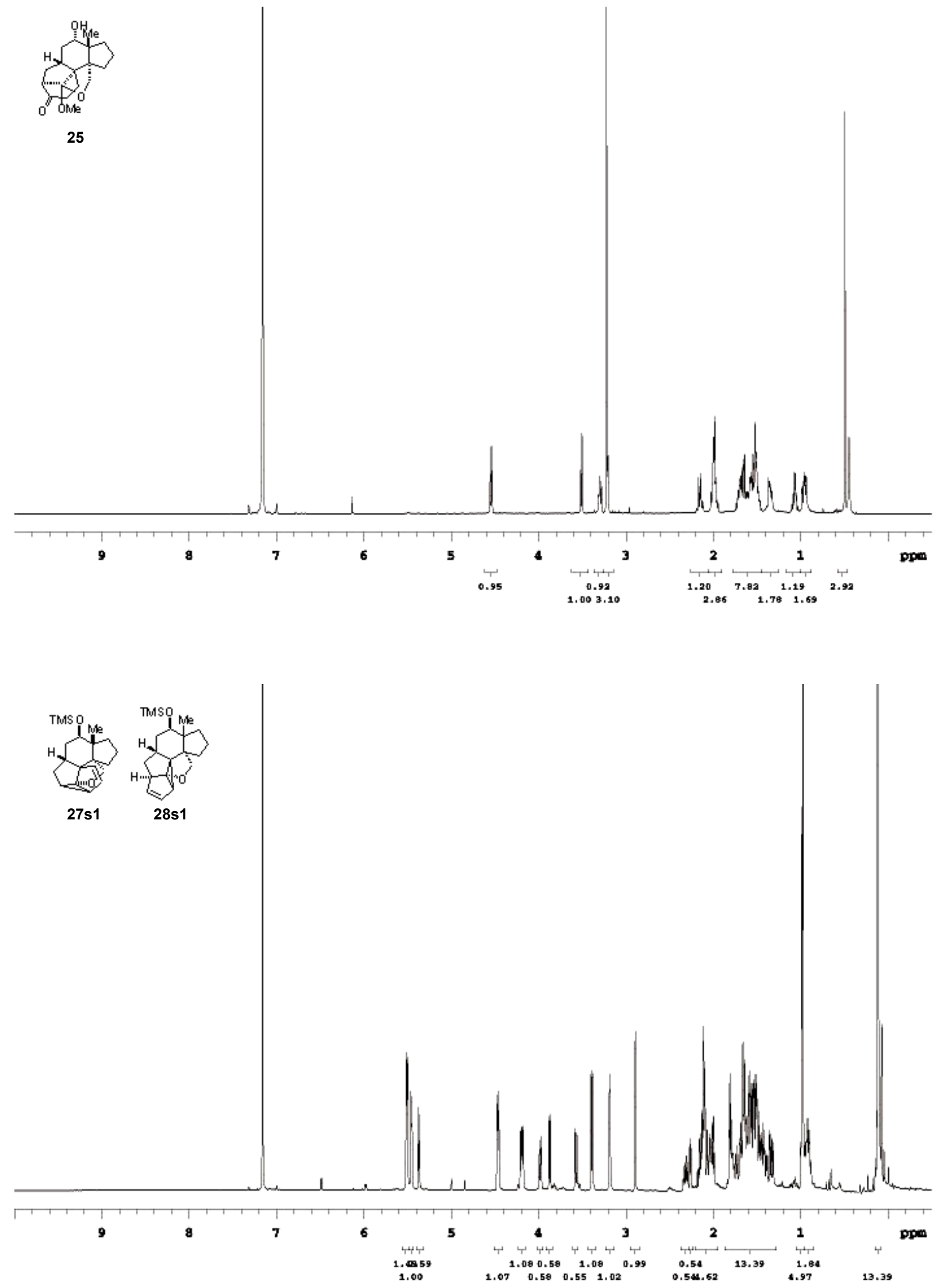

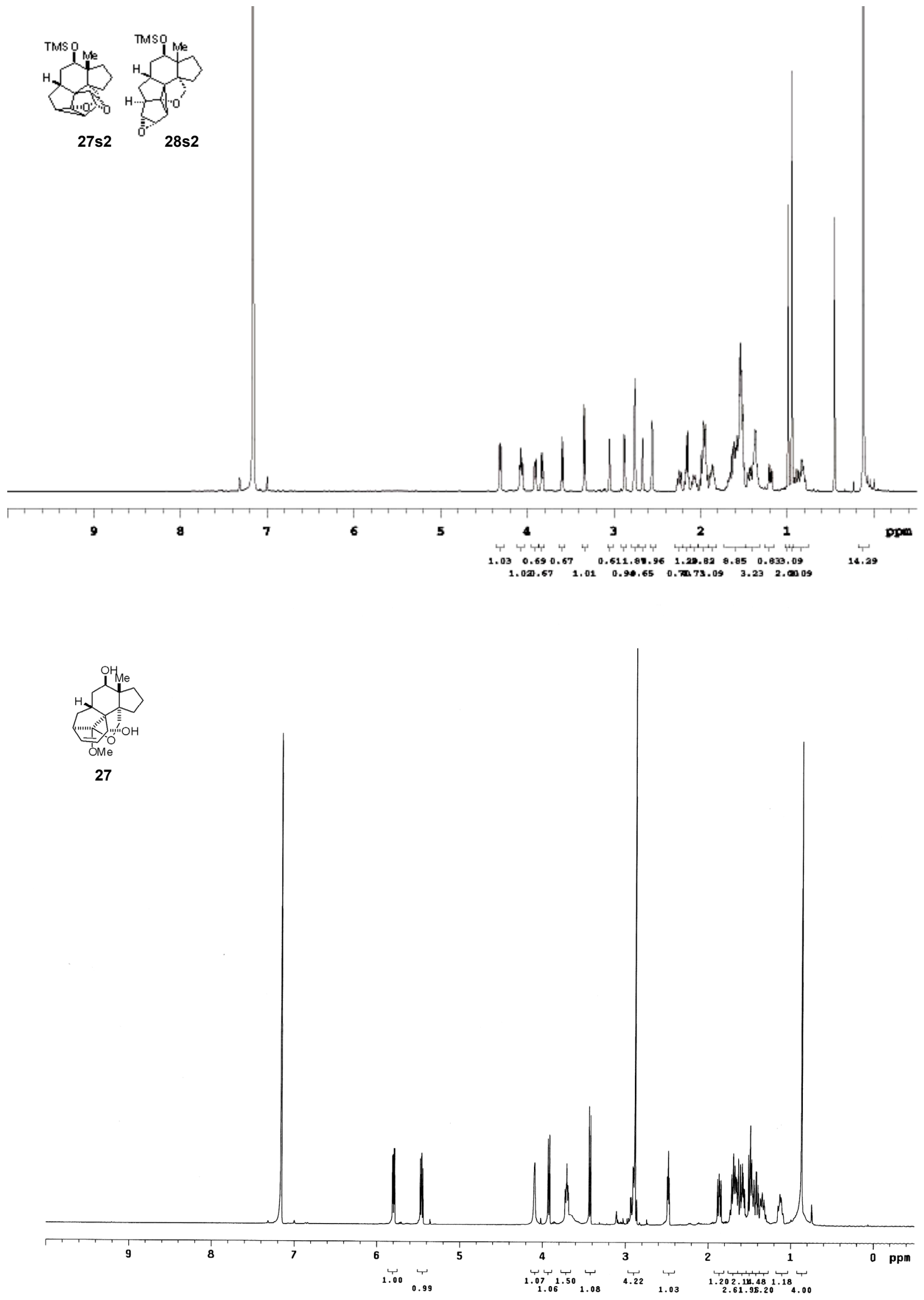

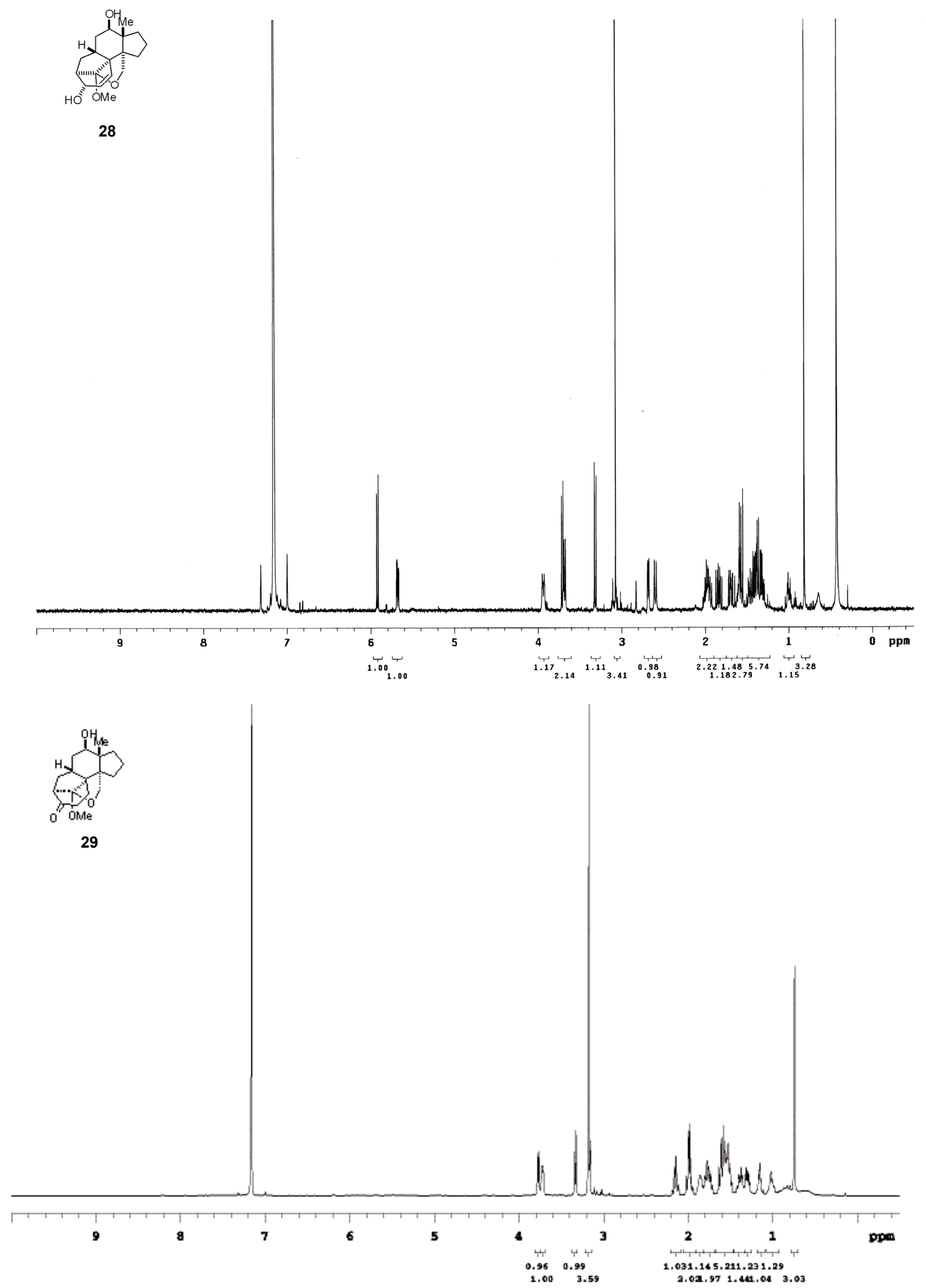

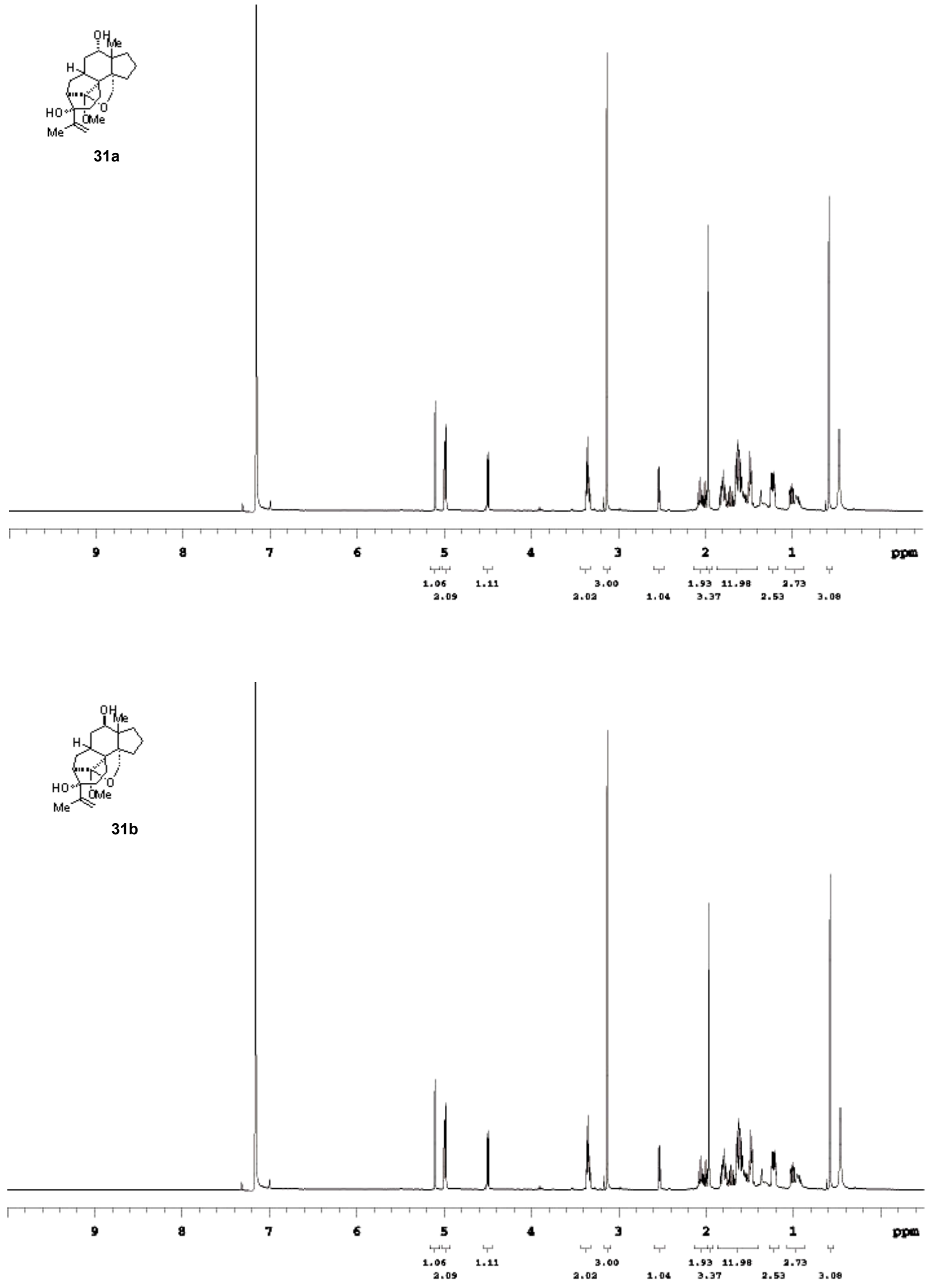

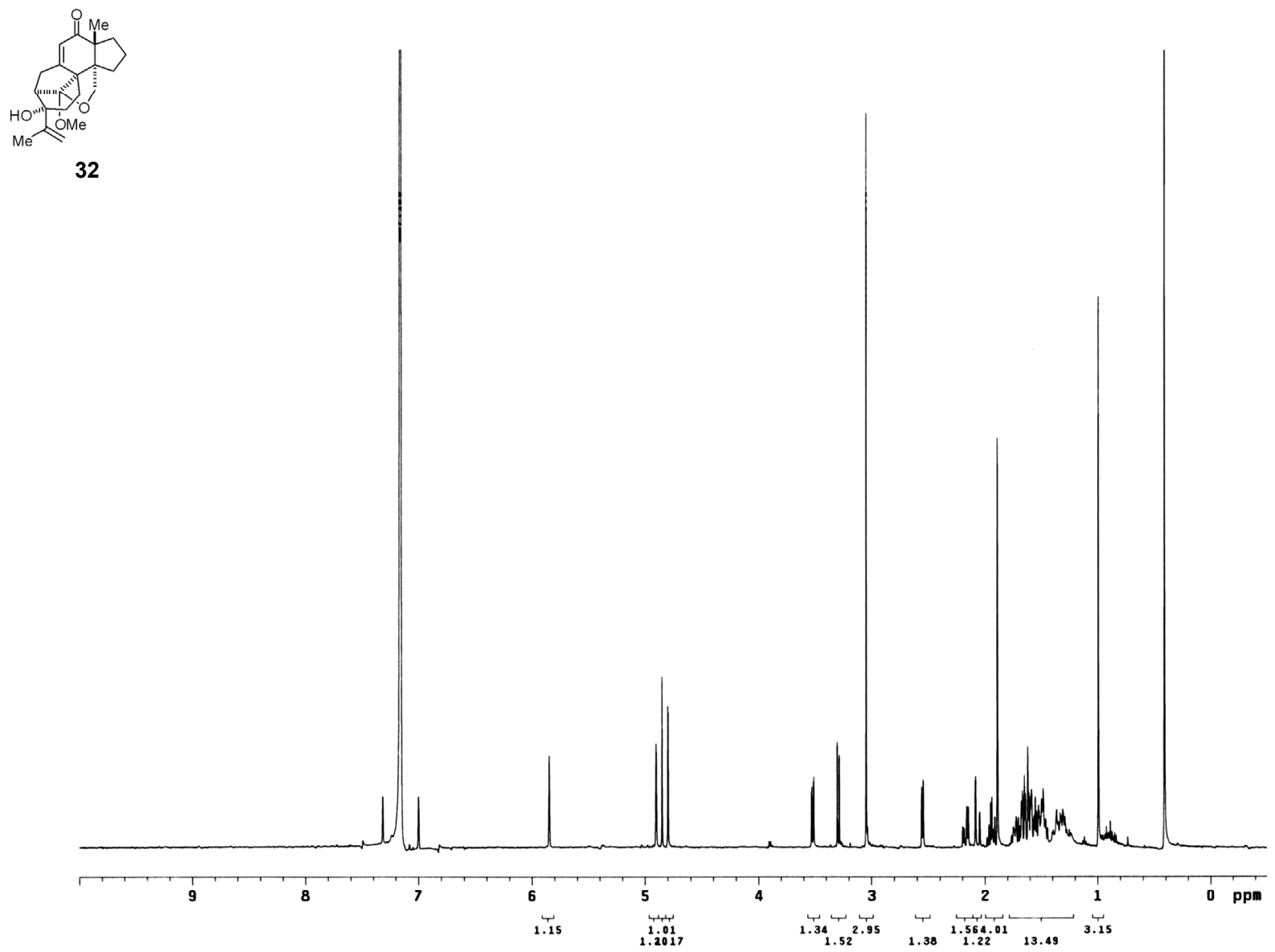